\title{
Mechanism of Tetramer Dissociation, Unfolding, and Oligomer Assembly of Pneumovirus M2-1 Transcription Antiterminators
}

\author{
Sebastián A. Esperante,* Damián Alvarez-Paggi, Mariano Salgueiro, and Gonzalo de Prat Gay*(i) \\ Protein Structure, Function and Engineering Laboratory, Fundación Instituto Leloir, IIBBA, Avenida Patricias Argentinas 435, 1405 \\ CABA, Argentina
}

\section{Supporting Information}

ABSTRACT: Among Mononegavirales, the Pneumovirus family stands out by its RNA polymerase processivity that relies on a transcription antiterminator, the M2-1 protein, which also plays a key role in viral particle assembly. Biophysical and structural evidence shows that this RNAbinding tetramer is strongly modulated by a $\mathrm{CCCH} \mathrm{Zn}^{2+}$ binding motif. We show that while the global dissociation/ unfolding free energy is $10 \mathrm{kcal} \mathrm{mol}^{-1}$, more stable for the respiratory syncytial virus M2-1, the human metapneumovirus (HMPV) counterpart shows a $7 \mathrm{kcal} \mathrm{mol}^{-1}$ higher intersubunit affinity. Removal of $\mathrm{Zn}^{2+}$ from both homologues leads to an apo-monomer of identical secondary structure that further undergoes a slow irreversible oligomerization. Mutation of the histidine residue of the $\mathrm{Zn}^{2+}$ motif to cysteine or alanine leads directly to large oligomers, strongly suggesting that metal coordination has an exquisite precision for modulating the quaternary arrangement. $\mathrm{Zn}^{2+}$ removal is very slow and requires subdenaturing concentrations of guanidine chloride, suggesting a likely local folding energy barrier. Exploring a broad combination of denaturant and ethylenediaminetetraacetic acid conditions, we showed that the metapneumovirus protein has to overcome a higher energy barrier to trigger $\mathrm{Zn}^{2+}$ removal-driven dissociation, in concordance with a slower dissociation kinetics. In silico modeling of open and close conformations for both M2-1 tetramers together with interaction energy calculations reveals that the gradual opening of protomers decreases the number of intersubunit contacts. Half of the interaction energy holding each protomer in the tetramer comes from the CCCH motif, while HMPV-M2-1 harbors additional contacts between the $\mathrm{CCCH}$ motif of one subunit and the core domain of a protomer located in trans, allowing the rationalization of the experimental data obtained. Overall, the evidence points at a key role of the $\mathrm{CCCH}$ motif in switching between structural and consequently functional alternatives of the M2-1 protein.

\section{INTRODUCTION}

Pneumoviridae family (Mononegaviridae order) includes human respiratory syncytial virus (RSV) and human metapneumovirus (HMPV), which are an important cause of acute lower respiratory tract infections and pneumonia mortality during the first year of life, especially in developing countries. ${ }^{1-3}$ Both viruses share similar structural genomic organizations and transcription/replication mechanisms. The single-stranded negative RNA genome tightly wrapped around the nucleocapsid $N$ protein is used as a template for transcription and replication. Genomic RNA replication is driven by the RNAdependent RNA polymerase $L$ protein together with the essential cofactor phosphoprotein $P$. An additional cofactor, the M2-1 protein unique to pneumovirus, is required for efficient subgenomic mRNA transcription. M2-1 acts as a transcription antiterminator and processivity factor, preventing the premature transcriptional termination of long mRNAs and improving the synthesis of polycistronic readthrough mRNAs. ${ }^{4}$ It also participates in post-transcriptional mRNA trafficking and transient accumulation of viral mRNAs in membraneless organelles, before releasing mRNAs into the cytosol for translation. ${ }^{5}$ Consequently, M2-1 is of significant interest for understanding Pneumovirus life cycle and infection. In addition to participating in mRNA transcription, it has been also associated with viral particle assembly, mediating the interactions between the $N$ nucleocapsid and Matrix $M$ protein. ${ }^{6}$ Moreover, electron cryotomographic characterization of RSV particles showed a layer of M2-1 between $M$ and $N$ ribonucleoprotein, supporting a role of M2-1 in viral morphogenesis. ${ }^{7}$ The multiple critical functions of M2-1 suggest the existence of alternative conformations, each compatible with specific functions. In this scenario, conformational switching should be finely tuned and could be susceptible to stimuli such as post-translational modifications, interactions, or changes occurring in the cellular environment such as $\mathrm{pH}$ or oxidative stress.

The X-ray crystallographic structures of M2-1 from RSV and HMPV have been obtained and both homologous proteins

Received: July 10, 2018

Accepted: October 17, 2018

Published: November 2, 2018 

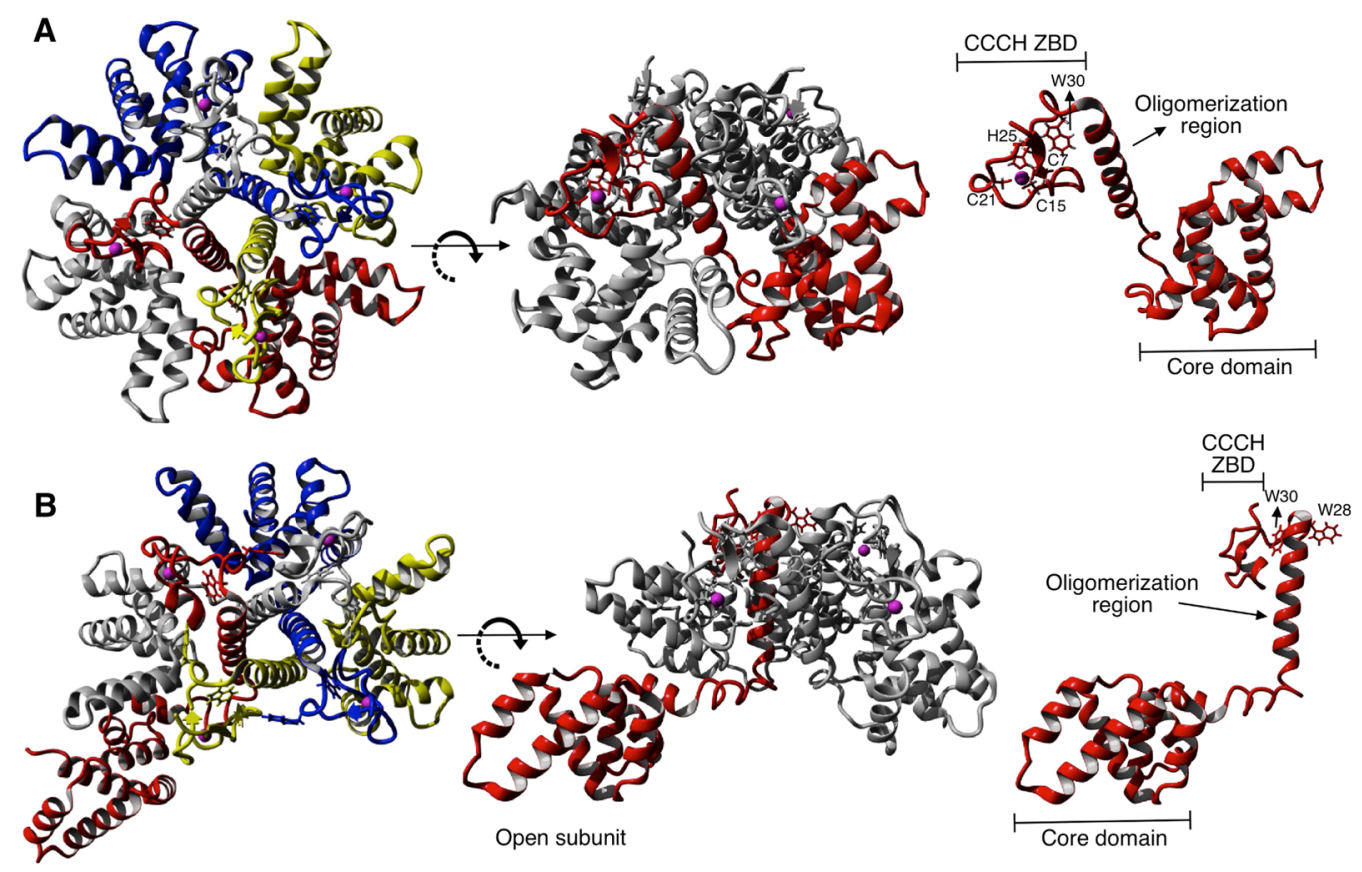

Figure 1. Structural arrangement of tetrameric RSV- and HMPV-M2-1 proteins. RSV-M2-1 (A) (protein data bank (PDB) id 4C3D) and HMPVM2-1 (B) (PDB id 4CS7). Left panel: tetramer with N-terminal face. Each subunit is presented in a different color code. Center panel: side face, with a single subunit colored red, to highlight the differences between the open and closed conformations. Right panel: a protomer of M2-1 with its structural regions indicated. $\mathrm{W} 30$ and $\mathrm{Zn}^{2+}$ coordinating residues, $\mathrm{C} 7, \mathrm{C} 15, \mathrm{C} 21$, and $\mathrm{H} 25$ are indicated. HMPV presents an additional $\mathrm{W}$ residue, W28, not present in RSV. All figures were prepared using YASARA. ${ }^{10}$

share a similar fold and structural arrangement, with a tetrameric quaternary structure (Figure 1)., ${ }^{8,9}$ The M2-1 protomer displays a modular architecture characterized by three distinct regions linked by unstructured or flexible sequences (Figure 1): the $\mathrm{N}$-terminal $\mathrm{CCCH} \mathrm{Zn}^{2+}$-binding domain (ZBD. residues 1-30), the tetramerization helix (residues 30-52), and the core domain (residues 66-170). A flexible linker (residues 53-65) that harbors phosphorylation sites connects the tetramerization helix with the core domain.

Although similar in overall fold and structure, with oligomerization driven by a four helix bundle at its center and stabilized by contacts between the $\mathrm{N}$-terminal ZBD interacting with adjacent protomers, the crystal structure of HMPV-M2-1 tetramer reveals an asymmetric unit with three protomers displaying a closed conformation and one with an open conformation. ${ }^{8}$ Interestingly, molecular dynamics simulations and small-angle X-ray scattering (SAXS) experiments of HMPV-M2-1 demonstrated a dynamic equilibrium between open and closed conformations in solution that is sensitive to the buffer composition. This equilibrium can be probed by the addition of denaturing agents such as guanidinium hydrochloride $(\mathrm{Gdm} . \mathrm{HCl})$ or the $\mathrm{Zn}^{2+}$-chelating agent ethylenediaminetetraacetic acid (EDTA) that tends to favor the open conformations, while RNA binding elicits the fully closed conformation. ${ }^{8}$ Crystal soaking experiments of HMPV-M2-1 with the DNA sequence AGTT revealed atomic contacts with the $\mathrm{ZBD}$ and the open-core domain, suggesting that both participate in the simultaneous recognition of RNA sequences. ${ }^{8}$ Altogether, these results show that the M2-1 tetramer constantly explores various conformations that are directly linked to physiological roles. The RNA-binding surface of RSV-M2-1 was located by mutational analysis, NMR chemical shift perturbation experiments, and RNA-binding assays, ${ }^{9,11}$ and was revealed to consist of a surface of positively charged residues within the core region of the tetramer and the $\mathrm{N}$ terminal residues $\mathrm{R} 3$ and $\mathrm{R} 4$.

RSV-M2-1, HMPV-M2-1, and other related proteins such as ebola VP30 contain a CCCH ZBD located upstream of the oligomerization helix that adopts a fold with low secondary structure content. $\mathrm{Zn}^{2+}$ binding to the RSV-M2-1 CCCH motif was shown to induce homointeractions to form a highly stable tetramer with RNA-binding ability, as well as processivity and antiterminator transcriptional activities. The tetrameric arrangement of RSV-M2-1 was shown to be essential to bind RNA molecules in a cooperative manner with high affinity and a loose sequence specificity. ${ }^{12}$ Moreover, RSV-M2-1 binds the tetrameric $P$ phosphoprotein with a singular tetramertetramer interface, ${ }^{13}$ suggesting that multiple RNA- or Pbinding sites are simultaneously interacting and outcompeting to exert its function. Consideration of its additional role during viral morphogenesis suggests that M2-1 adopts different conformations to perform its multiple activities interacting with different counterparts. The molecular determinants or signals switching these conformational/functional behaviors of M2-1 are still unknown, despite proposals for the $\mathrm{CCCH}$ motif of $\mathrm{M} 2-1$ as a $\mathrm{pH}$ sensor. ${ }^{14}$

In the present work, we assess the stability, alternative conformations, and oligomerization states of RSV- and HMPV-M2-1 proteins, analyzing the molecular basis of the thermodynamic and kinetic parameters derived for the studied transitions. We demonstrate that the $\mathrm{CCCH} \mathrm{Zn}^{2+}$ binding motif is not accessible in the fully closed conformation and that displacement toward the open tetramer conformations drastically increases its accessibility, rendering it susceptible to react with EDTA. We show that HMPV-M2-1 presents a higher energy barrier for triggering tetramer dissociation induced by $\mathrm{Zn}^{2+}$ removal, consistent with the slower 
dissociation kinetics observed. Performing in silico structural analysis allowed us to dissect the energetic contributions that stabilize the tetrameric arrangement in open and closed states. Altogether, these results lead us to propose a novel interaction surface in the HMPV tetramer, not found in RSV, which could explain the higher affinity and slower dissociation kinetics observed. Finally, we show that impairment of $\mathrm{Zn}^{2+}$ binding leads to the formation of higher-order oligomeric species, which could be crucial in viral assembly. These results highlight a plausible key role of the $\mathrm{CCCH}$ motif modulating structural/functional alternatives of M2-1 protein.

\section{RESULTS}

HMPV-M2-1 Tetramer Exhibited Higher Affinity Than the RSV-M2-1 Counterpart. To compare the conformational stability of the closely related HMPV- and RSV-M2-1 tetramers, we carried out $\mathrm{Gdm} . \mathrm{HCl}$-induced equilibrium denaturation experiments monitored by far-UV circular dichroism (CD) and tryptophan fluorescence emission measurements (Figure 2). At $10 \mu \mathrm{M}$ HMPV-M2-1 concentration, the secondary and tertiary structure signal changes are coupled showing stable baselines for the tetrameric (within 02.0 $\mathrm{M} \mathrm{Gdm} . \mathrm{HCl}$ ) and monomeric unfolded states, with a cooperative transition exhibiting an estimated denaturation midpoint $[D]_{50}$ of $2.5 \mathrm{M} \mathrm{Gdm.HCl}$ (Figure $2 \mathrm{~A}$ ). Denaturation curves performed at $1 \mu \mathrm{M}$ (Figure S1) shifted the transition midpoint to lower $\mathrm{Gdm} . \mathrm{HCl}$ concentrations, indicating that unfolding is coupled to tetramer dissociation. The far-UV CD and fluorescence denaturation curves at 1 and $10 \mu \mathrm{M}$ were globally fit to a three-state equilibrium model as was previously described for RSV-M2-1, ${ }^{15}$ and the thermodynamic parameters derived from the fits are summarized in Table 1 and Figure S1. Although the conformational stability of RSV-M2-1 has been previously studied, ${ }^{15}$ we performed additional equilibrium denaturation experiments of RSV-M2-1 for comparison purposes. Despite the fact that the global free energy of the dissociation/denaturation process $\left(\Delta G_{\mathrm{T}}{ }^{\mathrm{H} 20}\right)$ is higher for RSVM2-1, the HMPV-M2-1 tetramer displays higher intersubunit affinity $\left(\Delta G_{\text {DISS }}{ }^{\mathrm{H} 20}\right)$. The three-state model allows for the dissection of the global process into two equilibria. The first equilibrium is characterized by the free energy of dissociation $\left(\Delta G_{\text {DISS }}{ }^{\mathrm{H} 20}\right)$ from native tetramer to a partially folded monomeric intermediate, while the second one is described by the free energy of unfolding of the monomeric intermediate $\left(\Delta G_{\mathrm{U}-\mathrm{I}}^{\mathrm{H} 20}\right)$. Comparison of $\Delta G_{\mathrm{DISS}}{ }^{\mathrm{H} 20}$ between both variants show values of $42.3 \mathrm{kcal} \mathrm{mol}^{-1}$ for HMPV-M2-1 and $35.3 \mathrm{kcal}$ mol $^{-1}$ for RSV-M2-1, in agreement with previous results. ${ }^{15}$ These values correspond to dissociation constants $\left(K_{\mathrm{D}}\right)$ of $1.0^{-31}$ and $1.0^{-28} \mathrm{M}^{3}$ for HMPV- and RSV-M2-1 proteins, respectively. The $7.0 \mathrm{kcal} \mathrm{mol}^{-1}$ free-energy difference observed suggests either stronger or additional interactions stabilizing the HMPV-M2-1 tetrameric interface which are non-negligible.

$\mathrm{Gdm} . \mathrm{HCl}$ denaturation curves performed in the presence of $1 \mathrm{mM}$ EDTA exhibit uncoupling of $\mathrm{CD}$ and fluorescence signals (Figure 2B), as previously reported for RSV-M2-1, where $\mathrm{Zn}^{2+}$ removal induces tetramer dissociation to a stable apo-monomer. ${ }^{14}$ In contrast to RSV, the center of spectral mass (CSM) of the samples incubated with $1 \mathrm{mM}$ EDTA displayed two transitions. The first one takes place at $0-0.5 \mathrm{M}$ $\mathrm{Gdm} . \mathrm{HCl}$ with an initial CSM value of $28.400 \mathrm{~cm}^{-1}$, compatible with tetrameric species. Interestingly, this transition is also observed in the absence of EDTA, albeit with a smaller

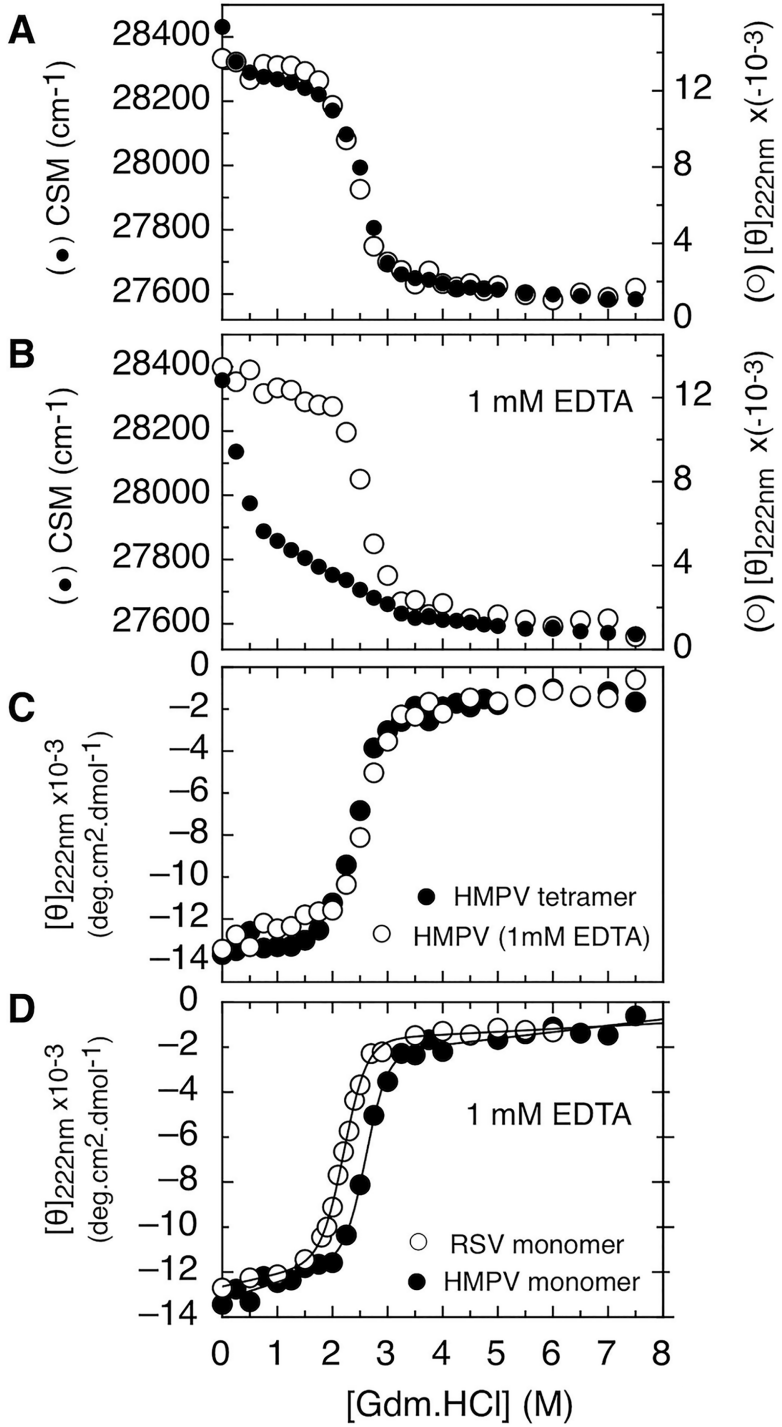

Figure 2. Gdm.HCl-induced equilibrium denaturation of HMPV-M21 protein at $\mathrm{pH}$ 7.0. Unfolding transition was followed by changes in molar ellipticity at $222 \mathrm{~nm}(\mathrm{O})$ and Trp center of spectral mass (CSM) (O). The samples at $10 \mu \mathrm{M}$ protein concentration were incubated in the absence (A) or presence (C) of $1 \mathrm{mM}$ EDTA. (B) Superimposition of the molar ellipticity changes at $222 \mathrm{~nm}$ in the absence (O) or presence (O) of $1 \mathrm{mM}$ EDTA. (D) Denaturation curves of HMPV (๑) and RSV (O) M2-1 proteins monitored by farUV-CD in the presence of $1 \mathrm{mM}$ EDTA. The CD data were fit to a two-state unfolding model (see Material and Methods), and the fits are plotted as solid lines. The $10 \mu \mathrm{M}$ samples were incubated for $24 \mathrm{~h}$ at $20{ }^{\circ} \mathrm{C}$ in $20 \mathrm{mM}$ sodium phosphate $\mathrm{pH} 7.0,0.3 \mathrm{M} \mathrm{NaCl}$ and the indicated $[\mathrm{Gdm} . \mathrm{HCl}]$.

amplitude that could be explained by changes in fluorescence of the additional Trp residue present in HMPV-M2-1, or alternatively, a small population undergoing an equilibrium, which is completely shifted in the presence of EDTA. The second transition occurs between 0.5 and $3.0 \mathrm{M} \mathrm{Gdm} . \mathrm{HCl}$ with low cooperativity and likely corresponds to changes in the tryptophan environment due to unfolding of the apomonomeric HMPV-M2-1. The CSM signal stabilizes at 3.0 $\mathrm{M} \mathrm{Gdm} . \mathrm{HCl}$, and this value $\left(27.600 \mathrm{~cm}^{-1}\right)$ corresponds to the unfolded monomer with its tryptophan residues completely exposed to the solvent. The secondary structure transitions for both HMPV-M2-1 tetramer and apo-monomer are similar 
Table 1. Thermodynamic Parameters Estimated from the Fit Using a Three-State Unfolding Model, Which Assumes the Population of Native Tetramer, a Partially Folded Monomeric Intermediate and Unfolded Monomer ${ }^{a}$

\begin{tabular}{|c|c|c|c|c|c|c|}
\hline \multirow[b]{2}{*}{$\mathrm{N}_{4} \Leftrightarrow 4 \mathrm{I} \Leftrightarrow 4 \mathrm{D}$} & \multicolumn{2}{|c|}{$\mathrm{N}_{4} \Leftrightarrow 4 \mathrm{D}$, global equilibrium } & \multicolumn{2}{|c|}{$\mathrm{N}_{4} \Leftrightarrow 4 \mathrm{I}$, equilibrium 1} & \multicolumn{2}{|c|}{$\mathrm{I} \Leftrightarrow \mathrm{D}$, equilibrium 2} \\
\hline & $\begin{array}{c}\Delta G_{\mathrm{T}}^{\mathrm{H} 20} \\
\left(\mathrm{kcal} \mathrm{mol}^{-1}\right)\end{array}$ & $\begin{array}{c}m_{\mathrm{T}} \\
\left(\mathrm{kcal} \mathrm{\textrm {mol } ^ { - 1 }} \mathrm{M}^{-1}\right)\end{array}$ & $\begin{array}{c}\Delta G_{\mathrm{DISS}}^{\mathrm{H} 20} \\
\left(\mathrm{kcal} \mathrm{mol}^{-1}\right)\end{array}$ & $\begin{array}{c}m_{\mathrm{DIS} S} \\
(\mathrm{kcal} \mathrm{mol} \\
\left.\mathrm{M}^{-1}\right)\end{array}$ & $\begin{array}{c}\Delta G_{\mathrm{U}-\mathrm{I}}^{\mathrm{H} 20} \\
\left(\mathrm{kcal} \mathrm{mol}^{-1}\right)\end{array}$ & $\left(\mathrm{kcal} \mathrm{\textrm {mol } _ { \mathrm { U } } ^ { - 1 }} \mathrm{M}^{-1}\right)$ \\
\hline$\Delta G_{\text {DISS }}{ }^{\mathrm{H} 20}$ & & & & & & \\
\hline$m_{\text {DISS }}$ & & & & & & \\
\hline HMPV-M2-1 & $46.8 \pm 0.5$ & $10.01 \pm 0.1$ & $42.3 \pm 0.1$ & $7.65 \pm 0.06$ & $1.2 \pm 0.1$ & $0.59 \pm 0.01$ \\
\hline RSV-M2-1 & $54.1 \pm 0.9$ & $14.08 \pm 0.06$ & $35.3 \pm 0.1$ & $5.52 \pm 0.02$ & $4.7 \pm 0.2$ & $2.14 \pm 0.01$ \\
\hline
\end{tabular}

${ }^{a}$ The equilibria and thermodynamic parameters that characterize each equilibrium are indicated. Global equilibrium thermodynamic parameters calculations were performed as follows:

$$
\begin{aligned}
& \Delta G_{\mathrm{T}}{ }^{\mathrm{H} 20}=\Delta G_{\mathrm{DISS}}{ }^{\mathrm{H} 20}+4 \Delta G_{\mathrm{U}-\mathrm{I}}{ }^{\mathrm{H} 20} \\
& m_{\mathrm{T}}=m_{\mathrm{DISS}}+4 m_{\mathrm{U}-\mathrm{I}}
\end{aligned}
$$

(Figure 2C), suggesting a stable fold of HMPV-M2-1 monomer and supporting the fact that Gdm.HCl-induced tetramer dissociation is coupled to monomer unfolding at this protein concentration.

It was shown that in the presence of subdenaturing $\mathrm{Gdm} . \mathrm{HCl}$ concentrations and EDTA, the major resulting species is the folded apo-monomer. ${ }^{14}$ Thus, we compared the conformational stability of RSV and HMPV-M2-1 apomonomers by fitting the $\mathrm{CD}$ data to a two-state model, considering an equilibrium folded to unfolded monomer transition (Figure 2D). While the apo-monomers show similar stability for each protein $\left(\Delta G_{\mathrm{U}-\mathrm{M}}{ }^{\mathrm{H} 20}=8.0 \mathrm{kcal} \mathrm{mol}^{-1}\right.$ for HMPV and $6.2 \mathrm{kcal} \mathrm{mol}^{-1}$ for RSV-M2-1), the HMPV monomeric folding intermediate species is less stable $\left(\Delta G_{\mathrm{U}-\mathrm{I}}^{\mathrm{H} 20}=1.2\right.$ vs $4.7 \mathrm{kcal} \mathrm{mol}^{-1}$ for RSV $)$. The lower stability of HMPV-M2-1 monomeric intermediate could be explained by the higher tetramer affinity, which couples dissociation to monomer unfolding at these protein concentrations. The thermodynamic parameters estimated from the fits are summarized in Table 2.

Table 2. Thermodynamic Parameters Estimated from the Fit Using Two-State Unfolding Model (See Materials and Methods) ${ }^{a}$

\begin{tabular}{ccc} 
& \multicolumn{2}{c}{$\mathrm{N} \Leftrightarrow \mathrm{D}$ equilibrium } \\
\cline { 2 - 3 } $\mathrm{N} \Leftrightarrow \mathrm{D}$, & $\Delta G_{\mathrm{U}-\mathrm{M}}{ }^{\mathrm{H}^{20}}\left(\mathrm{kcal} \mathrm{mol}^{-1}\right)$ & $m_{\mathrm{U}-\mathrm{M}}\left(\mathrm{kcal} \mathrm{mol}^{-1} \mathrm{M}^{-1}\right)$ \\
$\Delta G_{\mathrm{U}-\mathrm{M}}{ }^{\mathrm{H} 20}$ & & \\
$m_{\mathrm{U}-\mathrm{M}}$ & & \\
apo-HMPV-M2-1 & $8.0 \pm 0.9$ & $3.06 \pm 0.34$ \\
apo-RSV-M2-1 & $6.2 \pm 0.4$ & $2.80 \pm 0.16$
\end{tabular}

${ }^{a} \Delta G_{\mathrm{U}-\mathrm{M}}{ }^{\mathrm{H} 20}$ : global free energy of unfolding of the monomer.

HMPV-M2-1 Is More Resilient to Gdm.HCl-Induced Conformational Changes That Expose the $\mathrm{Zn}^{2+}$-Binding Domain. In addition to the usual use of tryptophan fluorescence to monitor tertiary structural changes, ${ }^{16}$ previous results from our lab show that the single Trp30 of RSV-M2-1 renders it an ideal probe for assessing its oligomerization state. $^{14}$ In the tetrameric state, Trp30 is buried in a hydrophobic environment showing a wavelength maximum of $325 \mathrm{~nm}$ (center of spectral mass, CSM $=29.400 \mathrm{~cm}^{-1}$ ), while in the folded apo-monomeric state, it shifts to $346 \mathrm{~nm}$ $\left(\mathrm{CSM}=28.000 \mathrm{~cm}^{-1}\right)$. To study the EDTA-induced dissociation of RSV- and HMPV-M2-1 tetramers, protein samples were incubated at two different concentrations ( 2 and $20 \mu \mathrm{M}$ ) in subdenaturing $\mathrm{Gdm} . \mathrm{HCl}$ conditions in the absence and presence of EDTA and subjected to fluorescence spectroscopy and dynamic light scattering (DLS) measurements. RSV-M2-1 showed a CSM of $29.400 \mathrm{~cm}^{-1}$ and diameters, as assessed by DLS, compatible with tetrameric species, at 0 and $0.8 \mathrm{M} \mathrm{Gdm} . \mathrm{HCl}$ (Figure 3A,B). The
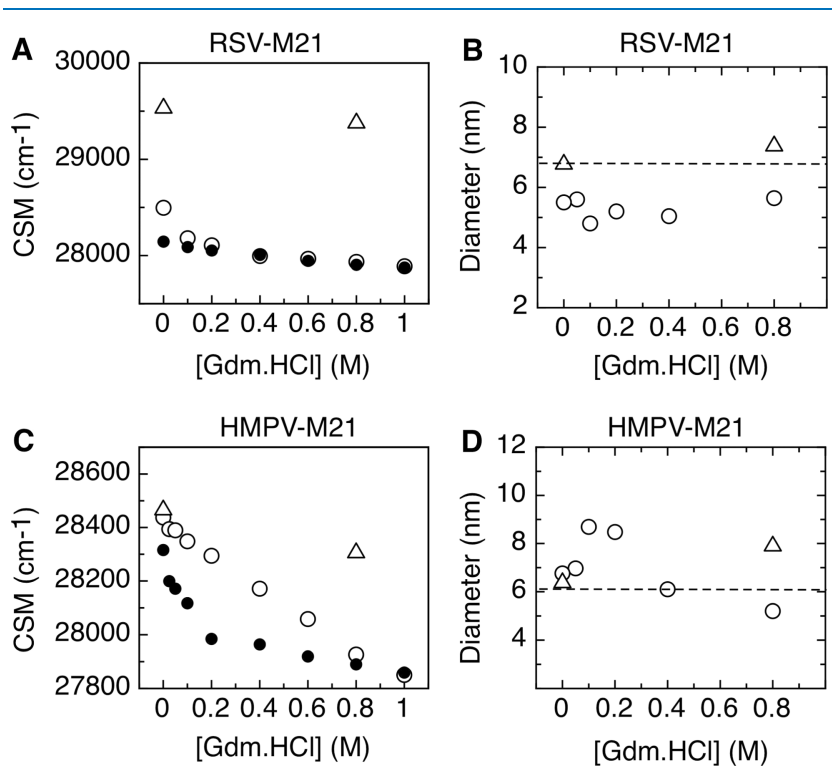

Figure 3. Protein concentration dependence on HMPV-M2-1 oligomerization state in the presence of EDTA. RSV- and HMPV$\mathrm{M} 2-1$ proteins at $2 \mu \mathrm{M}$ (filled symbols) or $20 \mu \mathrm{M}$ (open symbols) were incubated for $16 \mathrm{~h}$ at $20^{\circ} \mathrm{C}$ with increasing subdenaturing [Gdm. HCl] in the presence or absence of $1 \mathrm{mM}$ EDTA. RSV (A) and HMPV (C) M2-1 fluorescence center of spectra mass as a function of [Gdm.HCl] were determined in the presence (circles) or absence (triangles) of $1 \mathrm{mM}$ EDTA. RSV (B) and HMPV (D) M2-1 hydrodynamic diameters measured by DLS at $20 \mu \mathrm{M}$ as a function of [Gdm. $\mathrm{HCl}]$ in the presence (circles) or absence (triangles) of $1 \mathrm{mM}$ EDTA. Samples were incubated in $20 \mathrm{mM}$ sodium phosphate $\mathrm{pH}$ 7.0.

hydrodynamic diameter at $0.8 \mathrm{M} \mathrm{Gdm} . \mathrm{HCl}$ is compatible with that of an extended tetramer, as was reported for RSV- and HMPV-M2-1 in the presence of subdenaturing $\mathrm{Gdm} . \mathrm{HCl}$ concentrations. ${ }^{8,15}$ Incubation of RSV-M2-1 with 1 mM EDTA shifted the tryptophan emission CSM to $28.000 \mathrm{~cm}^{-1}$, suggesting a monomeric state along the $\mathrm{Gdm} . \mathrm{HCl}$ range tested (Figure 3A). DLS measurements of RSV-M2-1 samples 
further confirm its monomeric nature (Figure 3B). As the accuracy of DLS for determining the difference between the hydrodynamic diameter of tetramer and monomer is low, the results were confirmed by glutaraldehyde cross-linking and sodium dodecyl sulfate-polyacrylamide gel electrophoresis (SDS-PAGE), which showed that apo-monomers populate at low concentrations of $\mathrm{Gdm} . \mathrm{HCl}$ (Figure S2A).

In addition to the conserved buried Trp30, HMPV-M2-1 harbors an additional tryptophan at position $28(\operatorname{Trp} 28)$, which is partially solvent-exposed (Figure 1B). Both Trp residues are located within the $\mathrm{CCCH}$ motif and the $\alpha$-helical oligomerization region, and its fluorescence emission spectrum in the native state exhibits a maximum emission wavelength of $340 \mathrm{~nm}$ (CSM of $28.400 \mathrm{~cm}^{-1}$ ). HMPV-M2-1 was incubated at 2 and $20 \mu \mathrm{M}$ in increasing $\mathrm{Gdm} . \mathrm{HCl}$ concentrations with 1 mM EDTA. Surprisingly, tryptophan fluorescence CSM transitions differed between both protein concentrations suggesting that a concentration-dependent dissociation process is taking place (Figure 3C). DLS measurements of the HMPVM2-1 samples showed hydrodynamic diameters compatible with tetramers or extended tetramers from 0 to $0.2 \mathrm{M}$ $\mathrm{Gdm} . \mathrm{HCl}$, while the sample incubated at $0.8 \mathrm{M} \mathrm{Gdm} . \mathrm{HCl}$ exhibited a lower diameter, indicative of a monomeric species (Figure 3C,D). Glutaraldehyde cross-linking and SDS-PAGE confirmed that the major species populating the reaction at low $\mathrm{Gdm} . \mathrm{HCl}$ concentrations are tetramers, whereas monomers populate above $0.4 \mathrm{M} \mathrm{Gdm} . \mathrm{HCl}$ (Figure S2B).

To confirm these results, serial dilutions of RSV and HMPVM2-1 tetramers were performed in $0.1 \mathrm{M} \mathrm{Gdm} . \mathrm{HCl}$, in the absence or presence of $1 \mathrm{mM}$ EDTA and subjected to fluorescence spectroscopy. In the absence of EDTA, both RSVand HMPV-M2-1 samples showed CSM compatible with tetrameric species (Figure S3), whereas in the presence of EDTA, RSV-M2-1 showed monomer-compatible CSM values at all protein concentrations. In contrast, HMPV-M2-1 gradually shifted its CSM upon protein dilution, compatible with tetrameric and monomeric states, respectively (Figure S3).

The experiments described above were performed with a 50-500-fold molar excess of EDTA relative to the available $\mathrm{Zn}^{2+}$ linked to each monomer. To assess the effect of EDTA on the quaternary structure, RSV- and HMPV-M2-1 tetramers were incubated at a fixed subdenaturing $\mathrm{Gdm} . \mathrm{HCl}$ concentration with increasing EDTA and subjected to fluorescence spectroscopy after 24 and $48 \mathrm{~h}$ incubation. At low EDTA concentrations, a stable tetrameric CSM baseline was observed before the onset of a transition to a monomeric stable CSM baseline (Figure 4). The transition shape was highly dependent on $\mathrm{Gdm} . \mathrm{HCl}$ concentration and differed for both M2-1 homologue proteins. HMPV-M2-1 reached equilibrium after $24 \mathrm{~h}$ incubation at $1.5 \mathrm{M} \mathrm{Gdm} . \mathrm{HCl}$, showing a steep transition between the conformational states populating the equilibrium. Interestingly, RSV-M2-1 CSM signal reached equilibrium after $24 \mathrm{~h}$ incubation at $0.5 \mathrm{M} \mathrm{Gdm} . \mathrm{HCl}$, indicating that it required lower denaturant concentration to facilitate EDTA-mediated $\mathrm{Zn}^{2+}$ removal and the concomitant tetramer-to-monomer dissociation (Figure 4B).

We have previously shown that lower $\mathrm{pH}$ values result in RSV-M2-1 tetramer destabilization. ${ }^{15}$ Therefore, the experiments were also performed in the absence of $\mathrm{Gdm} . \mathrm{HCl}$ at $\mathrm{pH}$ 5.0. Under these conditions, RSV-M2-1 fluorescence emission spectra remained unchanged at all of the EDTA concentrations tested. DLS measurement of a RSV-M2-1 sample incubated at

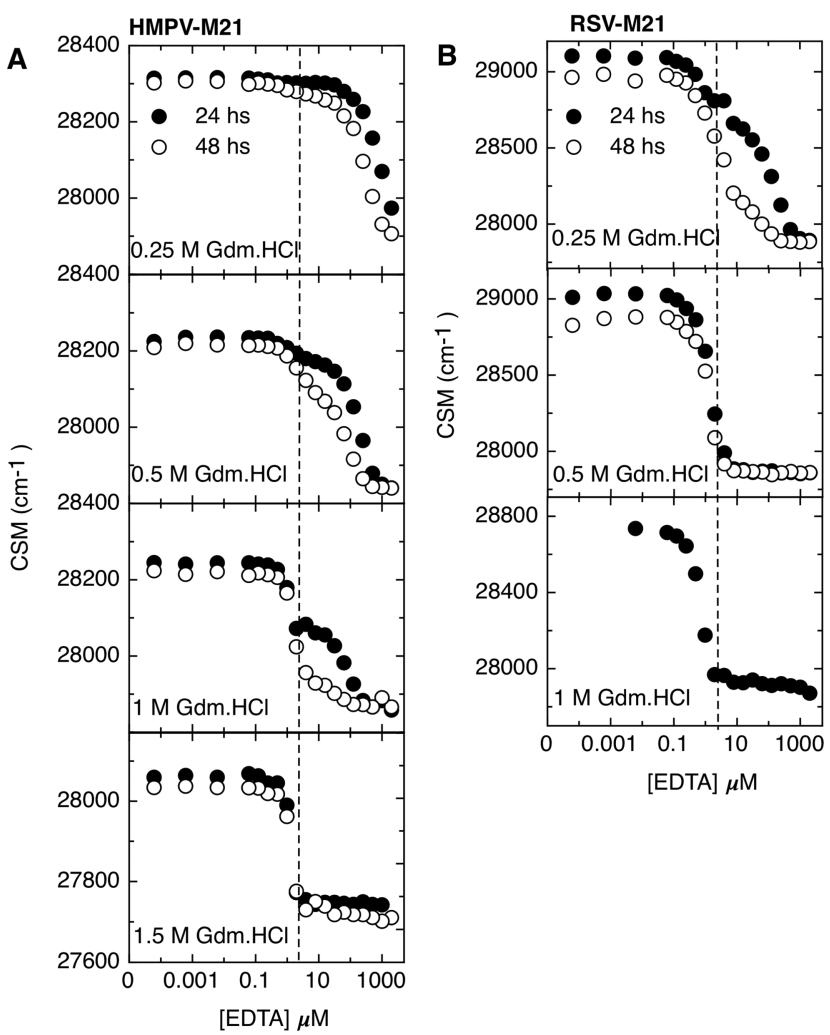

Figure 4. Influence of EDTA and subdenaturing [Gdm. HCl] on the oligomerization state of M2-1 homologous proteins; $2 \mu \mathrm{M}$ of HMPV (A) and RSV (B) M2-1 proteins were incubated for 24 and $48 \mathrm{~h}$ in 50 $\mathrm{mM}$ sodium phosphate $\mathrm{pH} 7.0$ containing different subdenaturing [Gdm.HCl] $(0.25 ; 0.5 ; 1.0$, and $1.5 \mathrm{M})$ and increasing EDTA concentrations (from 0 to $5 \mathrm{mM}$ ). After incubation, tryptophan fluorescence emission spectra were measured, and the center of spectral mass is represented as a function of EDTA concentration. The dashed line indicates the position of $2 \mu \mathrm{M}$ EDTA in the $x$ axis and the influence over the CSM signal at this fixed EDTA concentration depending on $[\mathrm{Gdm} . \mathrm{HCl}]$.

pH 5.0 with $1 \mathrm{mM}$ EDTA showed a hydrodynamic diameter of $37 \pm 12 \mathrm{~nm}$ (data not shown) with a CSM of $28.500 \mathrm{~cm}^{-1}$ (Figure S4), suggesting that the apo-monomer is not stable in solution in the absence of $\mathrm{Gdm} . \mathrm{HCl}$ and self-assembly of M2-1 into higher-order oligomeric species takes place. Interestingly, upon incubation of HMPV-M2-1 at $\mathrm{pH} 5.0$, fluorescence emission spectra showed an intensity transition in the EDTA range spanning $0.1-10 \mu \mathrm{M}$, suggesting that, below $0.1 \mu \mathrm{M}$ EDTA, the intensity signal corresponds to $\mathrm{Zn}^{2+}$-bound M2-1 species, while above $10 \mu \mathrm{M}$, the stable intensity is indicative of the apo-species (Figure S4). Considering that the first coordination sphere of $\mathrm{Zn}^{2+}$ ion is identical in both protein species, these results strongly hint at the $\mathrm{pH}$ modulation of the $\mathrm{CCCH}$ through the protein matrix acting as a second (and higher) coordination sphere. ${ }^{17}$ Several histidine residues proximal to coordinating cysteines in the $\mathrm{CCCH}$ binding motif of RSV-M2-1 not present in HMPV-M2-1 (Figure S4E) are likely candidates for exerting this $\mathrm{pH}$-dependent modulation. It seems likely that the additional HMPV-M2-1 Trp30 is able to sense $\mathrm{Zn}^{2+}$ binding to the $\mathrm{CCCH}$ motif. Incubation of $40 \mu \mathrm{M}$ HMPV-M2-1 at $\mathrm{pH} 5.0$, in the presence or absence of EDTA, leads to protein aggregation that precludes the determination of the hydrodynamic diameter of the apospecies. 
Mutations That Affect $\mathrm{Zn}^{2+}$ Coordination to $\mathrm{CCCH}$ Motif Lead to the Formation of Higher-Order ApoOligomeric RSV-M2-1 Species. $\mathrm{Zn}^{2+}$ removal mediated by EDTA leads to tetramer dissociation, yielding an apo-M2-1 monomer that is stabilized in solution with subdenaturing concentrations of $\mathrm{Gdm} . \mathrm{HCl}$. However, in the absence of denaturant, apo-monomers exhibit a strong tendency to form higher-order oligomers. We next assessed the structural and conformational effect of mutations in the $\mathrm{CCCH}$ motif that could hinder $\mathrm{Zn}^{2+}$ binding. RSV-M2-1 His residue 25, which is one of the $\mathrm{Zn}^{2+}$ coordinating residues, was mutated either to $\mathrm{Ala}$ or Cys. Size exclusion chromatography (SEC) in a Superdex 200 column showed that both mutants, namely, $\mathrm{H} 25 \mathrm{~A}$ and H25C RSV-M2-1, eluted at the void volume, indicating that the $\mathrm{CCCH}$ histidine mutations impaired tetrameric assembly and promoted the formation of higherorder oligomeric species (Figure 5A), with a hydrodynamic
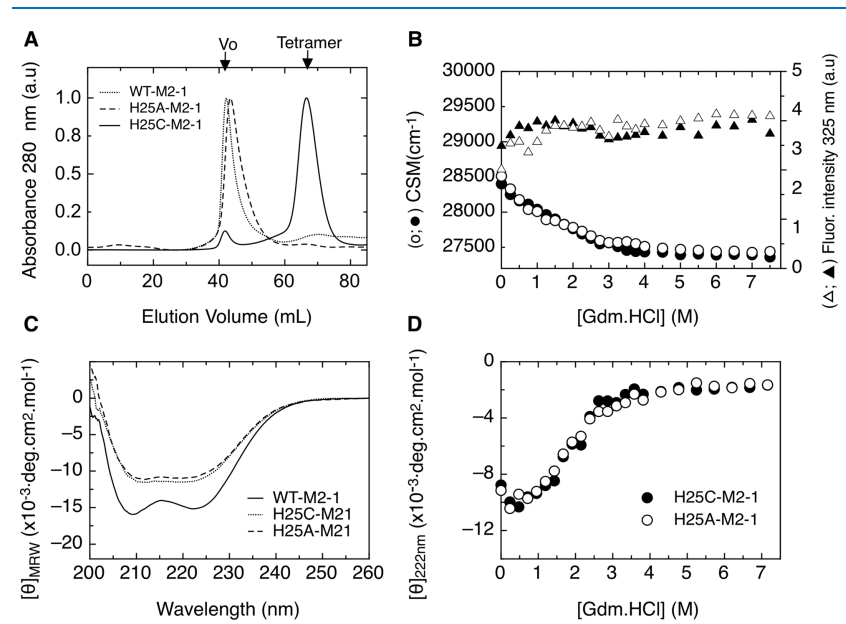

Figure 5. Characterization of RSV-M2-1 higher-order oligomeric species induced by $\mathrm{CCCH}$ mutations. (A) Size exclusion chromatography in a preparative grade Superdex 200 column of WT-, H25A-, and $\mathrm{H} 25 \mathrm{C}-\mathrm{M} 2-1$ proteins. The void volume ( $\mathrm{Vo}$ ) and the elution volume of WT-M2-1 tetramer are indicated. (B) Gdm.HCl-induced equilibrium denaturation of H25A- and H25C RSV-M2-1 proteins, followed by the changes in the Trp center of spectral mass (left axis, circles) and changes in the fluorescence emission intensity at $325 \mathrm{~nm}$ (right axis, triangles). Filled symbols (H25C-M2-1) and open symbols (H25A-M2-1). (C) Far-UV-CD spectra of WT-, H25A-, and H25C$\mathrm{M} 2-1$ proteins in $50 \mathrm{mM}$ sodium phosphate $\mathrm{pH} 7.0$ and $0.3 \mathrm{M} \mathrm{NaCl}$. The spectrum of each protein is indicated in the graph. (D) $\mathrm{Gdm} . \mathrm{HCl}$ equilibrium denaturation of H25A- (open symbol) and H25C(closed symbol) RSV-M2-1 proteins followed by the changes in molar ellipticity at $222 \mathrm{~nm}$.

diameter of $19 \pm 4 \mathrm{~nm}$. The amount of $\mathrm{Zn}^{2+}$ bound to each monomer was determined spectrophotometrically by the metallochromic indicator 4-(2-pyridylazo)resorcinol (PAR) (see Materials and Methods for details) ${ }^{15,18}$ and neither $\mathrm{H} 25 \mathrm{~A}$ nor $\mathrm{H} 25 \mathrm{C}$ displayed bound $\mathrm{Zn}^{2+}$, indicating that (i) a single substitution to Ala of the fourth coordinating residue impaired $\mathrm{Zn}^{2+}$ binding and (ii) most interestingly, the geometry of a CCCC motif in this protein context is not able to coordinate $\mathrm{Zn}^{2+}$, suggesting that a structural reorganization should take place for successful coordination.

To characterize the apo-RSV-M2-1 oligomers, we next studied the conformational stability of both mutants by Gdm.HCl-induced equilibrium denaturation experiments. The tryptophan CSM showed a gradual transition with low cooperativity from 0 to $3.0 \mathrm{M} \mathrm{Gdm} . \mathrm{HCl}$ (Figure 5B). The initial CSM value corresponded to the tryptophan environment of apo-RSV-M2-1 oligomeric species, and in contrast to the tetramer-to-monomer denaturation experiments, the fluorescence intensity did not change along the denaturation curve (Figure 5B). The far-UV CD spectra of the mutants at 0 $\mathrm{M} \mathrm{Gdm} . \mathrm{HCl}$ were slightly different related to wild-type tetrameric RSV-M2-1 (Figure 5C). The minima at 208 and $222 \mathrm{~nm}$ characteristic of rich $\alpha$-helical conformation decreased, suggesting a lower content of $\alpha$ helical structure of the oligomeric M2-1 species (Figure 5C). The conformational changes followed at $222 \mathrm{~nm}$ as a function of $\mathrm{Gdm} . \mathrm{HCl}$ concentration displayed a transition that starts at low denaturant concentrations with an endpoint at $2.5 \mathrm{M}$ $\mathrm{Gdm} . \mathrm{HCl}$ (Figure 5D). It is worth mentioning that the denaturation transition midpoint for both WT-RSV apomonomer $(10 \mu \mathrm{M})$ or tetramer $(2.5 \mu \mathrm{M})$ is $2.5 \mathrm{M} \mathrm{Gdm} . \mathrm{HCl}$ after a stable folded baseline. The absence of a stable folded baseline together with the fact that the molecule was fully unfolded at $2.5 \mathrm{M} \mathrm{Gdm} . \mathrm{HCl}$, strongly suggesting that the apomonomer, which self-assembles to yield higher-order M2-1 apo-oligomers, adopts a different fold.

M2-1 Tetramer Dissociation Kinetics Triggered by EDTA $\mathrm{Zn}^{2+}$ Removal. EDTA-mediated $\mathrm{Zn}^{2+}$ removal from peptides harboring ZBDs (CCCC or $\mathrm{CCHH}$ motifs) have been described to occur within milliseconds or seconds, with the formation of a ternary complex between EDTA, $\mathrm{Zn}^{2+}$ ion, and protein ZBD. ${ }^{19}$ RSV-M2-1 was incubated in the absence of denaturant with $5 \mathrm{mM}$ EDTA, and aliquots at selected time points were subjected to fluorescence spectroscopy and size exclusion chromatography. The $\mathrm{Zn}^{2+}$ content of the isolated species was determined by the colorimetric PAR-p-hydroxymercuriphenylsulfonate (PMPS) assay. ${ }^{18}$ Fluorescence measurements showed that the tryptophan CSM slowly decreased from 29.400 to $29.000 \mathrm{~cm}^{-1}$ along $5 \mathrm{~h}$ incubation, and samples subjected to SEC showed that within this time range, all of the samples remained in the tetrameric conformation (Figure S5). $\mathrm{Zn}^{2+}$ content determined from the purified tetramers after SEC revealed that up to $5 \mathrm{~h}$ incubation, every M2-1 monomer conserved a bound $\mathrm{Zn}^{2+}$ ion. These results hinted at a conformational barrier preventing EDTA from accessing the $\mathrm{CCCH}$ motif that hindered $\mathrm{Zn}^{2+}$ removal. Addition of $1.0 \mathrm{M}$ $\mathrm{Gdm} . \mathrm{HCl}$ shifted the fluorescence CSM to that compatible with monomeric species within $30 \mathrm{~min}$ incubation (Figure S5). Therefore, increasing concentrations of $\mathrm{Gdm} . \mathrm{HCl}$ were needed to facilitate EDTA accessibility, $\mathrm{Zn}^{2+}$ removal, and tetramer dissociation.

A fixed volume of EDTA was added to samples incubated at increasing $\mathrm{Gdm} . \mathrm{HCl}$ concentrations, and tetramer dissociation kinetics were followed by tryptophan fluorescence intensity changes (Figure 6A). No burst phase was observed for both tetramers upon the addition of EDTA by manual mixing (dead time $20 \mathrm{~s}$ ). The Trp fluorescence intensity decreased concomitantly with the exposure of the Trp residue to the solvent upon dissociation. As expected, at a fixed EDTA concentration, increasing subdenaturing concentrations of $\mathrm{Gdm} . \mathrm{HCl}$ accelerated the dissociation reaction, and interestingly, RSV-M2-1 tetramer dissociation occurred faster than HMPV-M2-1 at the same incubating condition. To extract quantitative data of dissociation kinetics, the decrease in fluorescence intensity was fit to an exponential decay model. RSV-M2-1 fluorescence intensity changes were best fit to a biexponential whereas HMPV-M2-1 fluorescence data fitted 


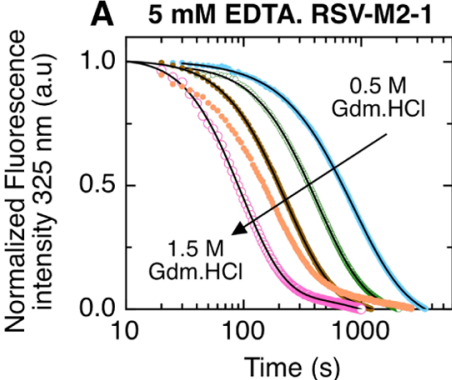

B 1.0 M Gdm.HCl. RSV-M2-1

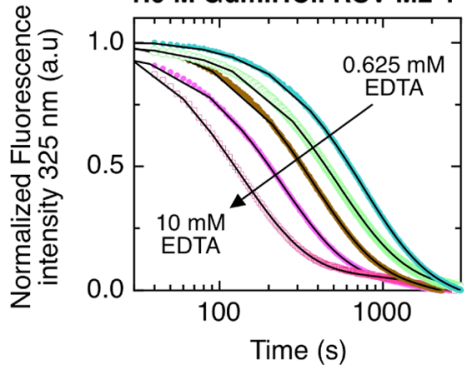

5 mM EDTA. HMPV-M2-1
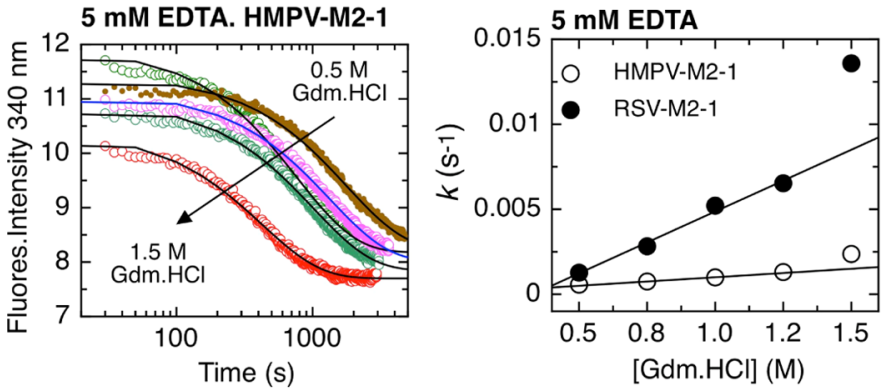

1.0 M Gdm.HCI. HMPV-M2-1
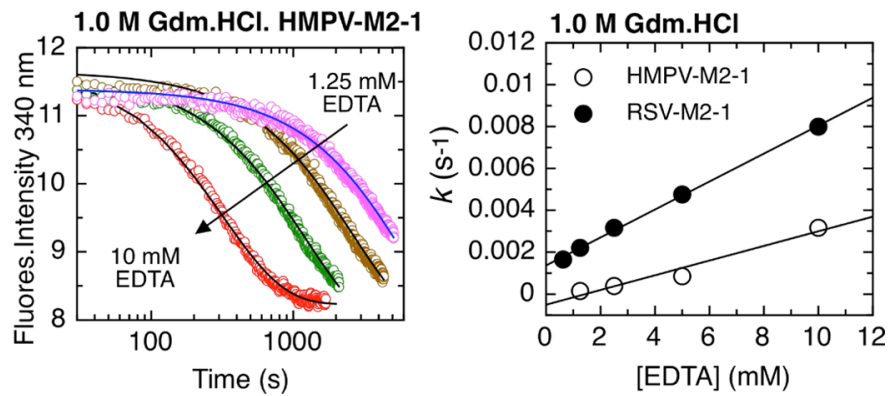

Figure 6. Dissociation kinetics of RSV- and HMPV-M2-1 proteins induced by EDTA and subdenaturing [Gdm.HCl]. (A) Fluorescence intensity changes produced after mixing $5 \mathrm{mM}$ EDTA and $2 \mu \mathrm{M} \mathrm{M} 2-1$ proteins in $50 \mathrm{mM}$ sodium phosphate $\mathrm{pH} 7.0$, containing increasing [Gdm.HCl] (0.5; $0.75 ; 1.0 ; 1.25$; and $1.5 \mathrm{M})$. Left panel: RSV-M2-1; middle panel: HMPV-M2-1; right panel: comparison of apparent rate constants as a function of [Gdm.HCl]. Linear fit: HMPV: $y=0.001 x-0.0005 ; R^{2}: 0.986$. RSV: $y=0.007 x-0.0024 ; R^{2}: 0.988$. The $k$ values at $1.5 \mathrm{M}$ Gdm.HCl were excluded from the linear fit as some extent of monomer unfolding occurs at this concentration. (B) Fluorescence intensity changes produced after mixing increasing concentrations of EDTA $(0.62 ; 1.25 ; 2.5 ; 5.0$; and $10 \mathrm{mM})$ and $\mathrm{M} 2-1$ proteins at $2 \mu \mathrm{M}$ in $50 \mathrm{mM}$ sodium phosphate pH 7.0 containing 1.0 M Gdm.HCl. Left panel: RSV-M2-1; middle panel: HMPV-M2-1; right panel: comparison of apparent rate constants as a function of EDTA concentrations at $1.0 \mathrm{M}$ Gdm.HCl. Linear fit: RSV: $y=0.0007 x-0.0014 ; R^{2}: 0.996$. HMPV: $y=0.0003 x-0.0005 ; R^{2}: 0.96$. The fluorescence changes along time were fit to a single exponential (HMPV-M2-1) or double exponentials (RSV-M2-1). The residuals from fits, fitted apparent rate constants, and amplitudes of each phase are shown in Figures S6 and S7.

well to a monoexponential decay model (Figure 6). Residuals to the fits and the estimated amplitudes and rate constants are shown in Figures S6 and S7. To compare the dissociation kinetics of both tetramers, the apparent rate decay constant of RSV- and HMPV-M2-1 $(k)$ were plotted as a function of $\mathrm{Gdm} . \mathrm{HCl}$ concentration. We observed a lineal dependency between $\mathrm{Gdm} . \mathrm{HCl}$ concentrations and apparent rate constants from 0.5 to $1.25 \mathrm{M} \mathrm{Gdm}$. HCl. Linear regression showed slopes of 0.007 for RSV and 0.001 for HMPV, reflecting a higher sensitivity to $\mathrm{Gdm} . \mathrm{HCl}$-induced dissociation at a fixed EDTA concentration (Figure 6A, right panel). At $1.5 \mathrm{M} \mathrm{Gdm.HCl}$ and $2 \mu \mathrm{M}$ protein concentration, dissociation to monomeric species is coupled to some extent of unfolding. Therefore, we excluded this point for the linear correlation analysis described above.

Based on these results, we speculate that subdenaturing $\mathrm{Gdm} . \mathrm{HCl}$ concentrations favor transitions to a partially or fully open conformation, exposing the $\mathrm{Zn}^{2+}$ bound to the $\mathrm{CCCH}$ motif. Therefore, M2-1 samples were incubated at a fixed subdenaturing $\mathrm{Gdm} . \mathrm{HCl}$ concentration and EDTA was added to trigger dissociation, followed by fluorescence intensity changes along time (Figure 6B). The reaction traces were fitted to biexponential (RSV-M2-1) or monoexponential functions (HMPV-M2-1) as described above. All of the phases depended linearly on EDTA concentrations in the tested range, and the slopes observed were 0.007 for RSV and 0.003 for HMPV. Assuming a similar $\mathrm{Zn}^{2+}$ binding affinity for both $\mathrm{CCCH}$ motifs, the lower HMPV-M2-1 dissociation rate could be explained as a higher energy barrier to overcome to expose the $\mathrm{CCCH}$ motif for $\mathrm{Zn}^{2+}$ removal. Or another possibility, in concordance with the results shown above, is a higher interaction energy stabilizing the tetramer interface of HMPV-M2-1

In Silico Modeling of Open and Closed M2-1 Tetramers Revealed Additional Contacts That Stabilize Further the HMPV-M2-1 Tetramer. Molecular dynamics simulations and SAXS experiments of HMPV-M2-1 tetramer revealed a rigid tetramerization domain and dynamic protomers fluctuating between open and closed states. ${ }^{8}$ To understand the structural and energetic contributions modulating the equilibrium between closed and open states, which might determine whether or not the tetramers expose the $\mathrm{CCCH} \mathrm{Zn}^{2+}$ binding motif, we constructed in silico molecular models of the following RSV and HMPV-M2-1 conformers: fully closed and one-, two-, three-, and four-open subunits (see Figure 8). The interaction network of each closed tetramer and the contacts that are lost in the fully open state were analyzed using CMView. ${ }^{20}$

Interestingly, we found that the HMPV-M2-1 tetramer presents additional interacting regions relative to RSV. The $\mathrm{CCCH} \mathrm{ZBD}$ of HMPV establishes contacts not only with the adjacent protomer but also with the core domain of the protomer located in trans. The interaction map of subunit $\mathrm{A}$ versus subunits $B, C$, and $D$ in the closed and fully open states is depicted in Figure 7A. Comparing HMPV- with RSV-M2-1, the region comprising the $\mathrm{CCCH}$ motif of subunit $\mathrm{C}$ displays additional contacts with the core domain of subunit A. A closeup inspection of this contact region reveals that the side chains of residues Cys 7, Cys 15, Cys 21, and His25 of subunit C interact with residues spanning from Val 108 to Asp 115 of 


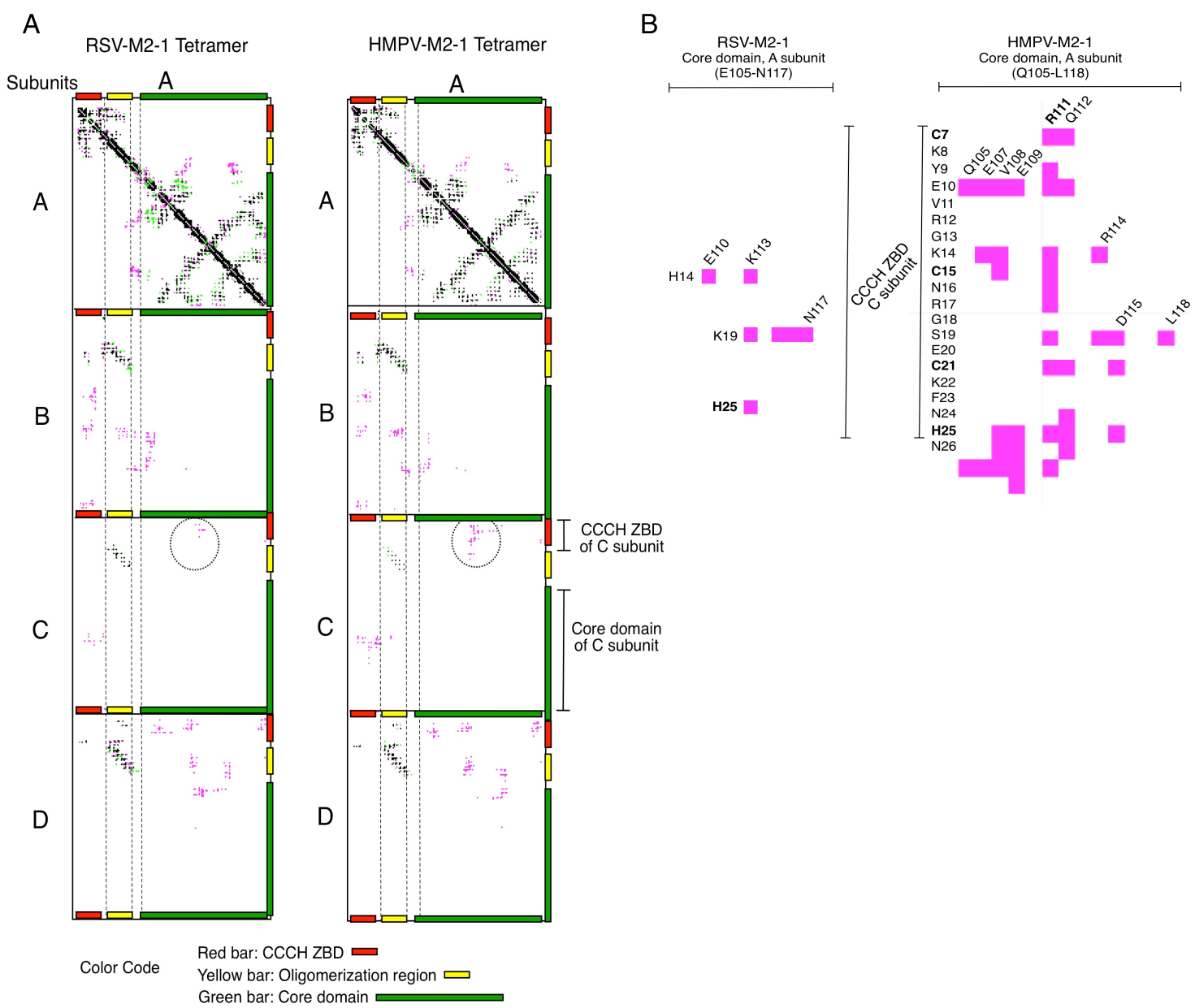

Figure 7. Comparison of the interaction maps between residues of the closed vs the fully open tetramer. (A) Interaction map of subunit $\mathrm{A}$ vs subunits A, B, C, and D is represented for RSV-M2-1 (left side) and HMPV-M2-1 (right side). The contacts present in both the closed and fully open states are shown in black. The contacts that are lost when the tetramer is fully open are shown in pink. Regions corresponding to the CCCH ZBD (red color bars), oligomerization region (yellow color bars), and core domain (green color bars) are indicated in each panel. The vertical dashed lines delineate the different regions of subunit A. Additional contacts observed for HMPV-M2-1 tetramer are circled in the C-A interaction panel. Interaction map was performed using the software tool CMView. ${ }^{20}$ (B) Close-up inspection of the interaction map showing contacts between the CCCH ZBD of subunit $\mathrm{C}$ and residues from the core domain of subunit A for RSV- (left panel) and HMPV- (right panel) M2-1 tetramers. Filled pink squares represent predicted contacts. The residues of the $\mathrm{CCCH}$ motif of subunit $\mathrm{C}$ are shown at the left-hand side of the contact map, while the residues spanning from 105 to 118 of the core domain of subunit A are indicated above the contact map.

subunit A (Figure 7B). A key residue of this interaction surface is Arg 111 that displays multiple contacts with all of the $\mathrm{Zn}^{2+}$ coordinating residues and some additional contacts within the $\mathrm{CCCH}$ motif. This striking difference between both tetramers may explain, in part at least, the differences in affinity and tetramer dissociation kinetics observed.

To gain further insight into the interaction network that stabilizes the open/closed tetrameric arrangement, we calculated the interaction energy of one protomer against the three remaining protomers using FoldX, ${ }^{21}$ and the results are summarized in Figure 8. For both closed tetramers, the interaction energies required to dissociate one protomer against the remaining subunits are similar for each subunit (Figure 8A,B). An in silico deletion of the $\mathrm{CCCH} \mathrm{Zn}{ }^{2+}$ binding domain allowed us to dissect the contribution of this motif and resulted in a 2 -fold decrease of the energy required to dissociate each closed protomer from the rest (Figure S8, closed tetramers). These results clearly indicate that almost half of the energy contribution that stabilizes one subunit in the closed state is provided by the ZBD. Comparison of the average energy required to dissociate one subunit from the rest, in the closed versus the open state, reveals that it is 3-fold higher for RSV-M2-1 (-98.82 vs $\left.-29.98 \mathrm{kcal} \mathrm{mol}^{-1}\right)$ and 1.8fold higher for HMPV-M2-1 (-81.93 vs $\left.-45.48 \mathrm{kcal} \mathrm{mol}^{-1}\right)$. In other words, the interaction energy calculations indicate that it is easier to dissociate an open RSV protomer rather than an open HMPV protomer. In both cases, deletion of the ZBD (or disruption as we hypothesize in the absence of $\mathrm{Zn}^{2+}$ ) decreases the interaction energy further (Figure S8). When one subunit is opened, the intersubunit contacts are lost while the oligomerization and ZBD domain of this subunit remain unaffected. The loss of intersubunit contacts decreases the interaction energy of the opened subunit almost 2-fold, whereas the absence of the ZBD decreases it further, strongly suggesting that an open apo-protomer would be a highly destabilized species. A single open protomer also decreases the interaction energies of its neighbors while the subunit located in trans is not affected. It is noteworthy that an open protomer increases the solvent surface accessible area of the ZBD of its adjacent protomer to an average of $500 \mathrm{~A}^{2}$ (see Table S1), and this could explain the EDTA accessibility and $\mathrm{Zn}^{2+}$ removal that was favored with $\mathrm{Gdm} . \mathrm{HCl}$. 

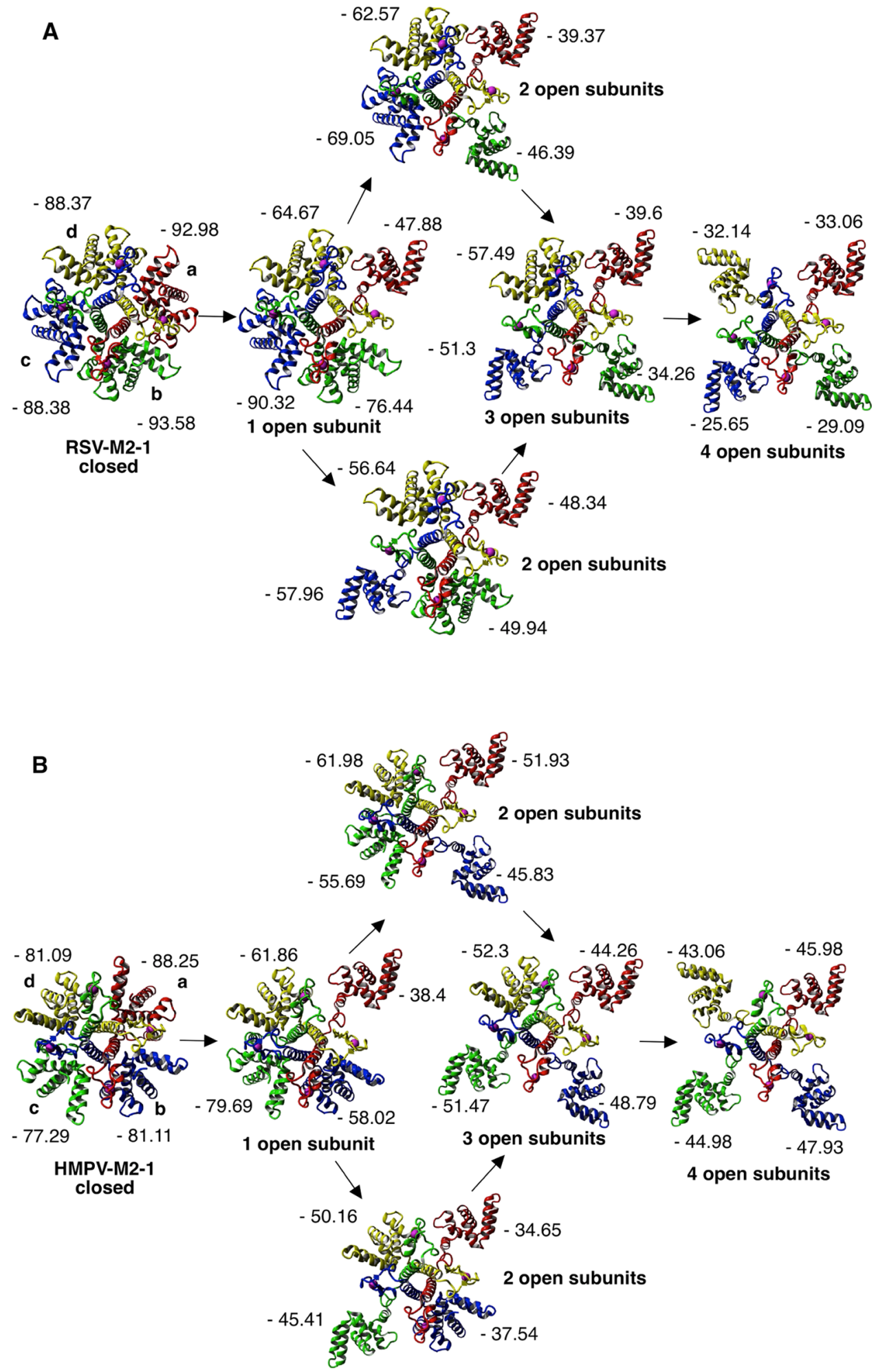

Figure 8. Interaction energies of each protomer against the remaining subunits of in silico modeled RSV- and HMPV-M2-1 tetramer conformers with open and closed subunits. RSV-M2-1 (A) and HMPV-M2-1 (B) tetramer conformers were constructed in silico, as detailed in Materials and Methods. The interaction energy of each protomer was calculated against the remaining three other subunits using FoldX. The interaction energy calculated for each protomer $\left(\mathrm{kcal} \mathrm{mol}^{-1}\right)$ is indicated close to the subunits depicted for each modeled tetramer. Red (a), green (b), blue (c), and yellow (d) subunits.

When two subunits are opened, either in cis or trans, they decrease not only the interaction energies of the open protomers but also those of the closed ones. The conformers with three- or four-open subunits show a decrease in the interaction energy of each subunit, and the absence of the ZBD

further destabilized the open subunits. 


\section{DISCUSSION}

Binding of zinc to proteins is an important regulatory mechanism for protein structure-function, and different $\mathrm{Zn}^{2+}$-binding domains have been involved in nucleic acidprotein and protein-protein interactions. ${ }^{22,23} \mathrm{RSV}$ and HMPV-M2-1 antiterminators contain a $\mathrm{CCCH}$ ZBD located upstream of the oligomerization region that stabilize the tetrameric arrangement in the holo-state. Tryptophan residues, located between the $\mathrm{CCCH}$ motif and the oligomerization region, are packed in a hydrophobic core, and when the tetramers dissociate, they become solvent-exposed leading to a red shift and fluorescence quenching. In the present work, we use the Trp fluorescence emission CSM to probe the tetrameric assembly and monitor equilibrium dissociation and dissociation kinetics triggered by a combination of EDTA and Gdm.HCl. The conformational behavior of RSV-M2-1 in solution was previously studied and consists of a tight tetramer with an estimated dissociation constant $\left(K_{\mathrm{D}}\right)$ of $10^{-28} \mathrm{M}^{3}$ that is highly sensitive to $\mathrm{pH}$ changes. Lowering the $\mathrm{pH}$ from 7.0 to 5.0 destabilizes the tetramer, and incubation of the protein at $\mathrm{pH} 3.0$ led to the formation of higher order soluble oligomeric species. $^{15} \mathrm{Gdm} . \mathrm{HCl}$ equilibrium denaturation curves of HMPV-M2-1 studied herein show a higher intersubunit affinity with a $K_{\mathrm{D}}$ of $10^{-31} \mathrm{M}^{3}$, suggesting either additional or stronger interactions stabilizing the HMPV-M2-1 tetrameric state. As was described for RSV-M2-1, the addition of EDTA led to uncoupling of signals probing the secondary and quaternary structural changes, due to tetramer dissociation to apo-monomeric species. However, higher subdenaturing $\mathrm{Gdm} . \mathrm{HCl}$ concentrations were needed to completely dissociate the HMPV tetramer in the presence of EDTA.

In many reported cases, binding of $\mathrm{Zn}^{2+}$ to a $\mathrm{CCCH}$ motif induces and stabilizes the native fold, and addition of EDTA leads to unfolding, such as human muscleblind-like protein. ${ }^{24}$ In both M2-1 tetramers, $\mathrm{Zn}^{2+}$ coordination was not necessary for the proper folding of the core domain but was essential to maintain the tetrameric arrangement. The measurement of the intrinsic $\mathrm{Zn}^{2+}$-binding affinity to a 59 residues peptide containing a $\mathrm{CCCH}$ motif revealed a dissociation constant of $1 \times 10^{-15} \mathrm{M}^{-1}$. ${ }^{25}$ Interestingly, this short peptide retains the same fold in the apo and holo states, thus allowing for the calculation of the free-energy contribution of $\mathrm{Zn}^{2+}$ binding to the $\mathrm{CCCH}$ motif at $\mathrm{pH} 7.0$ that is $-21.1 \mathrm{kcal} \mathrm{mol}^{-1}$. Considering this evidence in addition to our findings, binding of $\mathrm{Zn}^{2+}$ to the $\mathrm{CCCH}$ motif induces M2-1 tetramerization and it seems plausible that the energy cost of tetramerization $\left(-42.2 \mathrm{kcal} \mathrm{mol}^{-1}\right.$ for HMPV-M2-1) is provided by $\mathrm{Zn}^{2+}$ binding.

Supporting this notion, mutations of the His25 residue to alanine of the $\mathrm{CCCH}$ motif of RSV-M2-1 impaired $\mathrm{Zn}^{2+}$ binding, which led to the formation of higher-order oligomeric species (Figure 5). Previous mutational analysis of the $\mathrm{Zn}^{2+}$ coordinating residues of the RSV-M2-1 CCCH motif had shown that these residues were essential for antiterminator activity in minigenome assays and also impaired phosphorylation of M2-1. ${ }^{26}$ Moreover, mutations of $\mathrm{C} 21$ and $\mathrm{H} 25$ of the HMPV-M2-1 CCCH motif abolished $\mathrm{Zn}^{2+}$ binding activity and viruses harboring these mutations had delayed replication in cell culture and were highly attenuated in cotton rats. ${ }^{27} \mathrm{We}$ show that $\mathrm{Zn}^{2+}$-binding incapacity is coupled to the formation of higher-order M2-1 oligomeric species, which likely play a different role in infection rather than the tetrameric antiterminator RSV-M2-1 factor.

In the present work, a direct transfer of $\mathrm{Zn}^{2+}$ ion from M2-1$\mathrm{Zn}^{2+}$ to metal-free EDTA has been studied and the CSM provides a useful measure of the burial of Trp in the tetrameric state and an ideal probe for assessing dissociation to monomeric species along time. Several studies of $\mathrm{Zn}^{2+}$ finger motifs showed picomolar to femtomolar binding affinities. ${ }^{28}$ The kinetics of $\mathrm{Zn}^{2+}$ removal from two zinc-binding peptides (CCCC and CCHH motifs) by EDTA have been studied and showed a biphasic behavior. ${ }^{19}$ The first phase, independent of EDTA concentration, is completed within milliseconds, and the second phase, dependent on EDTA concentrations, proceeds in a time scale of seconds. The rapid association/ dissociation kinetics upon $\mathrm{Zn}^{2+}$ removal has been explained by the formation of a transient intermediary ternary $\mathrm{Zn}^{2+}$-bridged complex between EDTA and $\mathrm{Zn}^{2+}$-binding motifs. ${ }^{19}$ Thus, despite the fact that $\mathrm{Zn}^{2+}-\mathrm{ZBD}$ complexes are thermodynamically stable, with picomolar or femtomolar affinities, the complexes are kinetically unstable in competition situations, consistent with a rapid dissociation/association regulatory mechanism. ${ }^{19}$ When RSV-M2-1 tetramer is incubated with a large excess of EDTA in buffers without Gdm.HCl, the $\mathrm{Zn}^{2+}$ finger was not easily accessible to EDTA and we were able to isolate tetramers containing $\mathrm{Zn}^{2+}$ after several hours of incubation. Subdenaturing concentrations of $\mathrm{Gdm} . \mathrm{HCl}$ were thus needed to facilitate EDTA accessibility and $\mathrm{Zn}^{2+}$ removal, and thereby to promote dissociation of monomeric species. One possible explanation for this observation is based on a comprehensive structural characterization of HMPV-M2-1 using X-ray crystallography data, SAXS, and molecular dynamic simulations that showed the presence of HMPVM2-1 open conformers induced by increasing subdenaturant concentrations of $\mathrm{Gdm} . \mathrm{HCl}^{8}$ The polar nature of the interactions that stabilizes the core domain in a closed state is consistent with low concentrations of $\mathrm{Gdm} . \mathrm{HCl}$ shifting a dynamic equilibrium to the open state. ${ }^{8}$ We speculate that when the tetramer is fully closed, the $\mathrm{ZBD}$ is buried and not accessible to the solvent, while the addition of subdenaturing concentrations of $\mathrm{Gdm} . \mathrm{HCl}$ disrupts the polar interactions stabilizing the closed state and might favor the accessibility of EDTA to the $\mathrm{ZBD}$, leading to $\mathrm{Zn}^{2+}$ removal and tetramer dissociation. In this context, comparing RSV and HMPV-M2-1 tetramers, we observed a striking difference in the EDTAinduced dissociation kinetics and the concentration of Gdm. $\mathrm{HCl}$ needed to facilitate EDTA accessibility and $\mathrm{Zn}^{2+}$ removal (Figures 4 and 6). A dynamic conformational barrier shields the $\mathrm{CCCH}$ motif in both cases and higher concentration of $\mathrm{Gdm} . \mathrm{HCl}$ was required to favor EDTAmediated $\mathrm{Zn}^{2+}$ removal and dissociation of HMPV-M2-1. This could be in agreement with the higher tetramer affinity observed.

Tetramer dissociation kinetic studies showed an initial lag phase in the intensity signal, at a fixed EDTA concentration, which was dependent on $\mathrm{Gdm} . \mathrm{HCl}$ concentrations (Figure 6). M2-1 has the ability to explore in solution different open/ closed conformations, and this equilibrium is shifted to the open conformers adding increasing $\mathrm{Gdm} . \mathrm{HCl}$ concentrations. The open conformers increase the solvent surface accessible area, favoring EDTA-mediated $\mathrm{Zn}^{2+}$ removal that prompted dissociation. While it is not entirely known how many $\mathrm{Zn}^{2+}$ ions are necessary to remove to induce tetramer dissociation, when apo-RSV monomer is titrated with $\mathrm{Zn}^{2+}$ and followed by 
the changes in the fluorescence CSM, three $\mathrm{Zn}^{2+}$ ions per tetramer are required to get a CSM signal compatible with tetrameric species (unpublished data). Therefore, it is likely that when two $\mathrm{Zn}^{2+}$ ions are removed from an open tetramer conformation, the tetramer is thus highly destabilized and dissociate to folded apo-monomeric species.

The in silico tetrameric M2-1 models, displaying open and closed conformations, support the notion that an open conformation destabilizes the tetramer due to loss of intersubunit contacts. Furthermore, an open protomer increases the solvent surface accessible area of the ZBD of its adjacent protomer an average of $500 \mathrm{~A}^{2}$, and this likely exposes the $\mathrm{Zn}^{2+}$ bound to the $\mathrm{CCCH}$ motif to react with competitors. Modeling of the conformers not only allowed the dissection of the interaction energy contributions, but also highlighted the importance of an intact $\mathrm{ZBD}$ stabilizing the tetrameric arrangement. When the $\mathrm{ZBD}$ was deleted in silico, a quantitative estimate of the energetic contribution of this motif stabilizing each subunit in a tetrameric arrangement was possible. The deletion of the $\mathrm{ZBD}$ and how the absence of this motif destabilizes the tetramer is a plausible scenario of the disruption of the $\mathrm{CCCH}$ motif induced by $\mathrm{Zn}^{2+}$ removal. The crystallographic structures showed that the $\mathrm{CCCH}$ motif bound to $\mathrm{Zn}^{2+}$ folds with low secondary structure content forming a planar ring that interacts through opposite faces with the oligomerization domain and the adjacent protomer. ${ }^{8,9}$ The disruption of its planar structure by $\mathrm{Zn}^{2+}$ removal could lead to a structural rearrangement and loss of interaction surfaces stabilizing the tetrameric structure. Detailed inspection of the interaction network of the open and closed tetramers reveals additional interactions in HMPV-M2-1, which stabilizes the closed tetramer, and this could rationalize the difference in tetramer affinity observed and the higher energy barrier needed to overcome to trigger dissociation, which resulted in a slower dissociation kinetics. By in silico modeling, we found that the HMPV-M2-1 CCCH motif not only interacts with the adjacent protomer but also contacts the core domain of a protomer located in trans, and we propose Arg 111 of the core domain as a key residue of this interaction surface, which is less extensive in RSV-M2-1.

A dynamic open and close conformation of RSV and HMPV tetramers, modulated by different factors, such as ionic strength, molecular crowding, phosphorylation, ligand binding, or other unknown cellular signals, is compatible with the exposure of the key regulatory $\mathrm{CCCH}$ motif, which likely senses either $\mathrm{pH}$ or oxidative changes depending on the stage of viral infection. This, in turn could modulate the equilibrium between the processivity-antiterminator tetrameric M2-1 factor or higher-order M2-1 species that may be involved in late stages of infection, either in stress granules or during virion assembly. Further evidence would be needed to confirm this hypothesis, but the particular CCCH motif present in RSVand HMPV-M2-1 proteins and also VP30 ebola protein suggests the presence of a key module sensing cellular environment, leading to structural and functional changes of the proteins, depending on the stage of infection. Previous reports proposed that higher-order oligomeric forms of RSVor HMPV-M2-1 could be involved in viral morphogenesis ${ }^{7,8}$ and our findings support this hypothesis and shed light into a feasible mechanism driven by reversible $\mathrm{Zn}^{2+}$ binding to the $\mathrm{CCCH}$ motif, which allows $\mathrm{M} 2-1$ to interchange into structural/functional alternatives.

\section{MATERIALS AND METHODS}

Expression and Purification of M2-1 Proteins. The human RSV strain A and HMPV strain CAN97-83 M2-1 proteins were recombinantly expressed and purified as previously described for RSV-M2-1 protein (12). Briefly, nonfused versions of RSV and HMPV-M2-1 proteins were expressed in C41(DE3) cells harboring the open reading frames of each protein cloned into pRSETA plasmid. Expression was induced by adding $0.3 \mathrm{mM}$ isopropyl $\beta$-D-1thiogalactopyranoside at $\mathrm{OD}_{600 \mathrm{~nm}}$ of $0.6-0.8$, and cells were incubated overnight in a luria broth at $37{ }^{\circ} \mathrm{C}$. The soluble protein was precipitated with $40 \%$ ammonium sulfate, resuspended, dyalized, and purified with a combination of Heparin-Ceramic HyperD column and preparative gel filtration (S200) chromatographies. Protein concentration was determined spectrophotometrically using a molar extinction coefficient $\varepsilon_{280 \mathrm{~nm}}=13.200$ and $\varepsilon_{280 \mathrm{~nm}}=22.920 \mathrm{M}^{-1}$ $\mathrm{cm}^{-1}$ for RSV- and HMPV-M2-1 proteins, respectively. The protein concentration is expressed as monomer concentration.

Spectroscopic Determination of $\mathrm{Zn}^{2+}$ Bound to M2-1. Bound zinc was determined by spectrophotometric measurement of the metallochromic indicator 4-(2-pyridylazo)resorcinol (PAR) as previously described. ${ }^{18} \mathrm{~A}$ standard $\mathrm{Zn}^{2+}$ calibration curve was measured at $500 \mathrm{~nm}$ using $1-10 \mu \mathrm{M}$ zinc standard for atomic absorption spectroscopy (Sigma-Aldrich) in a solution containing $100 \mu \mathrm{M}$ PAR. Protein samples (2-8 $\mu \mathrm{M})$ were added to solutions containing $100 \mu \mathrm{M}$ PAR to measure weakly bound zinc. Tightly bound zinc was tested by the addition of $100 \mu \mathrm{M} p$-hydroxymercuriphenylsulfonate (PMPS).

Fluorescence Spectroscopy and Far-UV Circular Dichroism (CD). Tryptophan fluorescence emission spectra were recorded on a Jasco FP-6500 spectrofluorometer with an excitation wavelength of $295 \mathrm{~nm}$, and the emission spectrum was recorded from 310 to $450 \mathrm{~nm}$. Spectra were taken in a 5 $\mathrm{mm}$ path length quartz cuvette with excitation/emission slits of $5 / 5$. Fluorescence emission data were analyzed by subtracting the buffer background, and the center of spectral mass of the emission spectrum was quantified as follows:

$$
\operatorname{CSM}\left(\mathrm{cm}^{-1}\right)=\frac{\sum\left(v_{\mathrm{i}} \times F_{\mathrm{i}}\right)}{\sum F_{\mathrm{i}}}
$$

where $F_{\mathrm{i}}$ is the fluorescence emission at wavenumber $v_{\mathrm{i}}$, and the summation is carried out over the range of measured values of F. Far-UV CD measurements were conducted on a Jasco J810 spectropolarimeter using a Peltier temperature-controlled cell. Spectra were recorded between 200 and $260 \mathrm{~nm}$ at a standard sensitivity, at a rate of $200 \mathrm{~nm} \mathrm{~min}^{-1}$, a response time of $2 \mathrm{~s}, 0.2 \mathrm{~nm}$ data pitch, and a bandwidth of $2 \mathrm{~nm}$. All spectra were an average of at least four scans. Spectra were taken on a $0.1 \mathrm{~cm}$ path length cell. The ellipticity at $260 \mathrm{~nm}$ was subtracted from the other ellipticities as a baseline value. Raw data were converted to molar ellipticity using the following equation:

$$
[\theta] \mathrm{MRW}=\frac{\mathrm{deg}}{[\mathrm{c}] \times \text { \#bonds } \times L \times 10}
$$

where deg is the raw signal in millidegrees, [c] is protein concentration in molar units, \#bonds is the number of peptide bonds (number of amino acids -1 ), and $L$ is the path length in centimeter. 
Determination of the Oligomeric State of M2-1

Protein. Size exclusion chromatography and dynamic light scattering (DLS) measurements were used to determine the hydrodynamic volume of monomeric, tetrameric, and higherorder oligomeric species of M2-1 protein. Size exclusion chromatography was carried out on a preparative Superdex 200 column $(120 \mathrm{~mL})$ column (GE Healthcare). The void volume (Vo) and total volume $(\mathrm{Vo}+\mathrm{Vi})$ were determined by loading Blue Dextran and acetone, respectively. The buffers used in the runs are indicated in each case. The determination of the hydrodynamic size distribution of M2-1 oligomeric species was measured by dynamic light scattering (DLS) on a Zetasizer Nano S DLS device from Malvern Instruments (Malvern). The solutions were centrifuged at $14000 \mathrm{~g}$ for $10 \mathrm{~min}$ before measurements.

Guanidinium Chloride (Gdm. $\mathrm{HCl}$ )-Induced Denaturation Experiments. $\mathrm{Gdm} . \mathrm{HCl}$ denaturation curves were performed incubating RT M2-1 samples for $24 \mathrm{~h}$ at either 1 or $10 \mu \mathrm{M}$ in $50 \mathrm{mM}$ sodium phosphate $\mathrm{pH} 7.0$ containing increasing concentrations of $\mathrm{Gdm} . \mathrm{HCl}$. Denaturation curves at $10 \mu \mathrm{M}$ protein concentration were also performed in the presence of $1 \mathrm{mM}$ EDTA. After incubation, the samples were measured by far-UV CD and fluorescence spectroscopy at 20 ${ }^{\circ} \mathrm{C}$.

Data Analysis and Fittings of Gdm. $\mathrm{HCl}$ Denaturation Curves. Gdm.HCl-induced denaturation curves of HMPVand RSV-M2-1 proteins were fitted to a three-state equilibrium model, as previously detailed for RSV-M2-1 protein. ${ }^{15}$ The species that populate the equilibrium are the native tetramer $\left(\mathrm{N}_{4}\right)$, monomeric intermediate $(\mathrm{I})$, and denatured monomer (D). $K_{\mathrm{DISS}}$ is the tetramer dissociation constant, and $K_{\mathrm{U}-\mathrm{I}}$ is the unfolding constant of the monomeric intermediate

$$
\mathrm{N}_{4} \stackrel{K_{\mathrm{DISS}}}{\longleftrightarrow} 4 \mathrm{I} \stackrel{K_{\mathrm{U}-\mathrm{I}}}{\longleftrightarrow} 4 \mathrm{D}
$$

The free energy of each transition was considered to depend linearly on $\mathrm{Gdm} . \mathrm{HCl}$ concentration and is related to theequilibrium constants $K_{\mathrm{DISS}}$ and $K_{\mathrm{U}-\mathrm{I}}$ by

$$
\begin{aligned}
K_{\text {DISS }} & =\mathrm{e}^{-\left(\Delta G_{\mathrm{DISS}}^{\mathrm{H} 20}-m_{\mathrm{DISS}} \times[\mathrm{Gdn} . \mathrm{Cl}]\right) / R T} ; K_{\mathrm{U}-\mathrm{I}} \\
& =\mathrm{e}^{-\left(\Delta G_{\mathrm{U}-\mathrm{I}}^{\mathrm{H} 20}-m_{\mathrm{U}-\mathrm{I}} \times[\mathrm{Gdn} . \mathrm{Cl}]\right) / R T}
\end{aligned}
$$

where $R$ is the gas constant; $T$ is the temperature; $K_{\mathrm{DISS}}, K_{\mathrm{U}-\mathrm{I}}$, $\Delta G_{\text {DISS }}{ }^{\mathrm{H} 20}$, and $\Delta G_{\mathrm{U}-\mathrm{I}}^{\mathrm{H} 20}$ are the equilibrium constants and free energies of dissociation and monomeric intermediate unfolding, respectively; and the $m$-values, $m_{\mathrm{DISS}}$ and $\mathrm{m}_{\mathrm{U}-\mathrm{I}}$, are the denaturant dependence of the free energy of dissociation and unfolding, respectively. Far-UV CD and fluorescence CSM equilibrium denaturation data performed at 1 and $10 \mu \mathrm{M}$ monomer concentration were globally fitted to a three-state model to estimate the thermodynamic parameters involved.

When $1 \mathrm{mM}$ EDTA is added to the denaturation reaction, M2-1 dissociates to apo-monomeric species that is stabilized in solution with low concentrations of $\mathrm{Gdm}$. HCl. Therefore, the $\mathrm{CD}$ denaturation data collected in the presence of $1 \mathrm{mM}$ EDTA correspond to the secondary structural changes of monomer unfolding. The CD denaturation data collected at 10 $\mu \mathrm{M}$ monomer concentration was fitted to a two-state unfolding model that considers the equilibrium native $(\mathrm{N})$ to unfolded (U) monomer. ${ }^{14}$

$$
\mathrm{N} \stackrel{K_{\mathrm{U}-\mathrm{M}}}{\longleftrightarrow} \mathrm{D}
$$

where $K_{\mathrm{U}}$ is the unfolding constant that is related to the free energy of unfolding by

$$
K_{\mathrm{U}-\mathrm{M}}=\mathrm{e}^{-\left(\Delta G_{\mathrm{U}-\mathrm{M}}^{\mathrm{H} 20}-m \times[\mathrm{Gdn} \cdot \mathrm{Cl}]\right) / R T}
$$

where $R$ is the gas constant; $T$ is the temperature; $K_{U}$ and $\Delta G_{\mathrm{U}-\mathrm{M}}{ }^{\mathrm{H} 20}$ are the equilibrium unfolding constant and free energy of unfolding, respectively; and $m$ is the denaturant dependence of the free energy of dissociation. For further details of the three-state and two-state models used, see refs 13, 14. Fitting was carried out using the software program Profit (Quantum Soft, Uetikon am See, Switzerland) to obtain parameters and their standard deviations. It is worth mentioning that the free energy of unfolding of the apomonomer $\left(\Delta G_{\mathrm{U}-\mathrm{M}}{ }^{\mathrm{H} 20}\right)$ is a global unfolding process that includes the free energy of unfolding of the monomeric intermediate described above $\left(\Delta G_{\mathrm{U}-\mathrm{I}}^{\mathrm{H} 20}\right)$.

Kinetic of Tetramer Dissociation Triggered by EDTA. M2-1 protein samples at $2 \mu \mathrm{M}$ monomer concentration were incubated in $50 \mathrm{mM}$ sodium phosphate $\mathrm{pH} 7.0$ containing subdenaturing concentrations of $\mathrm{Gdm} . \mathrm{HCl}$ (indicated in each case). Thryptophan emission fluorescence changes at $325 \mathrm{~nm}$ (RSV-M2-1) or $340 \mathrm{~nm}$ (HMPV-M2-1) were followed along time upon the addition of different concentrations of EDTA. The Trp residues are located in an hydrophobic environment in the tetrameric state and exposed to the solvent upon dissociation, with a red shift and decrease in the fluorescence intensity. The fluorescence traces along time were fitted to mono- or biexponential decay models

$$
\Delta r=A_{1} \mathrm{e}^{k_{\mathrm{obs}_{1}} \cdot t}+A_{2} \mathrm{e}^{k_{\mathrm{obs}_{2}} \cdot t}+c \cdot t
$$

where $A_{1}$ and $A_{2}$ are the amplitudes and $k_{\mathrm{obs}_{1}}$ and $k_{\mathrm{obs}}$ are the observed rate constants. The signal drift was taken into account by a linear time-dependent term (c).

In Silico Modeling of RSV- and HMPV-M2-1 Open/ Closed Tetrameric Conformers. The primary structure of RSV-M2-1 strain A2 was used to model with SWISSMODEL $^{29,30}$ the fully closed RSV-M2-1 tetramer using as template the pdb 4C3B.1.a. The tetrameric structure of HMPV-M2-1 was modeled by structural homology with pdb 4SC7.1.a. The resulting HMPV-M2-1 tetrameric structure contains one open protomer with its core domain (B subunit) packed against the adjacent closed protomer (D subunit). We selected the AC dimer to model a closed symmetric HMPVM2-1 tetramer using visual molecular dynamics (VMD) ${ }^{31}$ and perform an energy minimization of the resulting closed tetramer using the YASARA force field. ${ }^{32}$ Based on the crystallographic structure of the open protomer (B), we changed the torsion angle using $\mathrm{VMD}^{31}$ to avoid steric clashes with the adjacent protomers and performed an energy minimization of the open subunit using YASARA force field. $^{32}$ We perform structural alignment of residues $1-50$ (that includes the ZBD and oligomerization domain) between the open protomer and each of tetramer subunits to position the open protomer into the coordinates of the tetrameric assembly. We modeled six conformers for each tetramer (see Figure 8) and repair the structures using the FoldX repair command. ${ }^{21}$ The interaction energies of each protomer against the remaining three protomers ( $\mathrm{A}$ vs $\mathrm{BCD}$; $\mathrm{B}$ vs $\mathrm{ACD}$; $\mathrm{C}$ vs $\mathrm{ABD}$; and $\mathrm{D}$ vs $\mathrm{ABC}$ ) were calculated using the FoldX AnalyseComplex command. ${ }^{21}$ The results obtained are summarized in Figure 8. 
Deletions of the $\mathrm{N}$ terminal 30 residues (Met1_Trp30del) were performed in silico over the four subunits of each pdb structure modeled to eliminate the ZBD contribution for the tetramer stability. All of the conformers have an intact oligomerization (residues 32-49) and core regions (residues 69-172). Therefore, the interactions that stabilize the $\mathrm{N}$ terminal deleted tetramers result from the oligomerization domain contacts and interprotomer contacts. The resulting tetrameric open/closed conformers lacking the ZBDs were used to calculate the interaction energies of each protomer against the three remaining protomers without the contribution of the ZBD. The FoldX AnalyseComplex was used to calculate the interaction energy of each protomer, and the results are summarized in Figure 8.

\section{ASSOCIATED CONTENT}

\section{S Supporting Information}

The Supporting Information is available free of charge on the ACS Publications website at DOI: 10.1021/acsomega.8b01600.

Global fitting of denaturation data; influence of $\mathrm{Gdm} . \mathrm{HCl}$ and EDTA; dilution-induced tetramer dissociation; influence of $\mathrm{pH}, \mathrm{Gdm} . \mathrm{HCl}$, and EDTA; slow RSV-M2-1 tetramer dissociation kinetics; parameters and residuals of HMPV-M2-1 and RSV-M2-1 dissociation kinetics; interaction energies (Figures $\mathrm{S} 1-\mathrm{S} 8$ ); and solvent accessible surface area (Table S1) (PDF)

\section{AUTHOR INFORMATION}

\section{Corresponding Authors}

*E-mail: sesperante@leloir.org.ar (S.A.E.).

*E-mail: gpg@leloir.org.ar (G.d.P.G.).

\section{ORCID}

Gonzalo de Prat Gay: 0000-0001-5748-6863

\section{Funding}

This work was supported by grant: PICT-2016-4605 from ANPCyT. M.S. holds a graduate fellowship from Consejo Nacional de Investigaciones Científicas y Técnicas (CONICET); S.A.E., D.A.-P. and G.d.P.G. are CONICET Career Investigators.

\section{Notes}

The authors declare no competing financial interest.

\section{ACKNOWLEDGMENTS}

The authors thank Fernando Goldbaum for assistance with DLS measurements and Salvador Ventura for assistance and helpful discussions with in silico modeling and interaction energy calculations using FoldX.

\section{REFERENCES}

(1) Scheltema, N. M.; et al. Global respiratory syncytial virusassociated mortality in young children (RSV GOLD): a retrospective case series. Lancet Global Health 2017, 5, e984-e991.

(2) Shi, T.; et al. Global, regional, and national disease burden estimates of acute lower respiratory infections due to respiratory syncytial virus in young children in 2015: a systematic review and modelling study. Lancet 2017, 390, 946-958.

(3) Panda, S.; Mohakud, N. K.; Pena, L.; Kumar, S. Human metapneumovirus: review of an important respiratory pathogen. Int. J. Infect. Dis. 2014, 25, 45-52.
(4) Fearns, R.; Collins, P. L. Role of the M2-1 transcription antitermination protein of respiratory syncytial virus in sequential transcription. J. Virol. 1999, 73, 5852-5864.

(5) Rincheval, V.; Lelek, M.; Gault, E.; Bouillier, C.; Sitterlin, D.; Blouquit-Laye, S.; Galloux, M.; Zimmer, C.; Eleouet, J. F.; RameixWelti, M. A. Functional organization of cytoplasmic inclusion bodies in cells infected by respiratory syncytial virus. Nat. Commun. 2017, 8, No. 563.

(6) Li, D.; Jans, D. A.; Bardin, P. G.; Meanger, J.; Mills, J.; Ghildyal, R. Association of respiratory syncytial virus $M$ protein with viral nucleocapsids is mediated by the M2-1 protein. J. Virol. 2008, 82, $8863-8870$.

(7) Liljeroos, L.; Krzyzaniak, M. A.; Helenius, A.; Butcher, S. J. Architecture of respiratory syncytial virus revealed by electron cryotomography. Proc. Natl. Acad. Sci. U.S.A. 2013, 110, 1113311138.

(8) Leyrat, C.; Renner, M.; Harlos, K.; Huiskonen, J. T.; Grimes, J. $M$. Drastic changes in conformational dynamics of the antiterminator M2-1 regulate transcription efficiency in Pneumovirinae. elife 2014, 3, No. e02674.

(9) Tanner, S. J.; Ariza, A.; Richard, C. A.; Kyle, H. F.; Dods, R. L.; Blondot, M. L.; Wu, W.; Trincao, J.; Trinh, C. H.; Hiscox, J. A.; Carroll, M. W.; Silman, N. J.; Eleouet, J. F.; Edwards, T. A.; Barr, J. N. Crystal structure of the essential transcription antiterminator M2-1 protein of human respiratory syncytial virus and implications of its phosphorylation. Proc. Natl. Acad. Sci. U.S.A. 2014, 111, 1580-1585.

(10) Krieger, E.; Vriend, G. YASARA View - molecular graphics for all devices - from smartphones to workstations. Bioinformatics 2014, 30, 2981-2982.

(11) Blondot, M. L.; Dubosclard, V.; Fix, J.; Lassoued, S.; AumontNicaise, M.; Bontems, F.; Eleouet, J. F.; Sizun, C. Structure and functional analysis of the RNA- and viral phosphoprotein-binding domain of respiratory syncytial virus M2-1 protein. PLoS Pathog. 2012, 8, No. e1002734.

(12) Molina, I. G.; Esperante, S. A.; Marino-Buslje, C.; Chemes, L. B.; de Prat-Gay, G. Cooperative RNA Recognition by a Viral Transcription Antiterminator. J. Mol. Biol. 2018, 430, 777-792.

(13) Esperante, S. A.; Paris, G.; de Prat-Gay, G. Modular unfolding and dissociation of the human respiratory syncytial virus phosphoprotein $\mathrm{p}$ and its interaction with the $\mathrm{m}(2-1)$ antiterminator: a singular tetramer-tetramer interface arrangement. Biochemistry 2012, $51,8100-8110$.

(14) Esperante, S. A.; Noval, M. G.; Altieri, T. A.; de Oliveira, G. A.; Silva, J. L.; de Prat-Gay, G. Fine modulation of the respiratory syncytial virus M2-1 protein quaternary structure by reversible zinc removal from its Cys(3)-His(1) motif. Biochemistry 2013, 52, 67796789.

(15) Esperante, S. A.; Chemes, L. B.; Sanchez, I. E.; de Prat-Gay, G. The respiratory syncytial virus transcription antiterminator $\mathrm{M}(2-1)$ is a highly stable, zinc binding tetramer with strong $\mathrm{pH}$-dependent dissociation and a monomeric unfolding intermediate. Biochemistry 2011, 50, 8529-8539.

(16) Lakowicz, J. R. Principles of Fluorescence Spectroscopy; Springer US: Baltimore, 2006.

(17) Alvarez-Paggi, D.; Abriata, L. A.; Murgida, D. H.; Vila, A. J. Native $\mathrm{Cu}(\mathrm{A})$ redox sites are largely resilient to $\mathrm{pH}$ variations within a physiological range. Chem. Commun. 2013, 49, 5381-5383.

(18) Hunt, J. B.; Neece, S. H.; Ginsburg, A. The use of 4-(2pyridylazo)resorcinol in studies of zinc release from Escherichia coli aspartate transcarbamoylase. Anal. Biochem. 1985, 146, 150-157.

(19) Heinz, U.; Kiefer, M.; Tholey, A.; Adolph, H. W. On the competition for available zinc. J. Biol. Chem. 2005, 280, 3197-3207.

(20) Vehlow, C.; Stehr, H.; Winkelmann, M.; Duarte, J. M.; Petzold, L.; Dinse, J.; Lappe, M. CMView: interactive contact map visualization and analysis. Bioinformatics 2011, 27, 1573-1574.

(21) Schymkowitz, J.; Borg, J.; Stricher, F.; Nys, R.; Rousseau, F.; Serrano, L. The FoldX web server: an online force field. Nucleic Acids Res. 2005, 33, W382-W388. 
(22) Hall, T. M. Multiple modes of RNA recognition by zinc finger proteins. Curr. Opin. Struct. Biol. 2005, 15, 367-373.

(23) Gamsjaeger, R.; Liew, C. K.; Loughlin, F. E.; Crossley, M.; Mackay, J. P. Sticky fingers: zinc-fingers as protein-recognition motifs. Trends Biochem. Sci. 2007, 32, 63-70.

(24) Park, S.; Phukan, P. D.; Zeeb, M.; Martinez-Yamout, M. A.; Dyson, H. J.; Wright, P. E. Structural Basis for Interaction of the Tandem Zinc Finger Domains of Human Muscleblind with Cognate RNA from Human Cardiac Troponin T. Biochemistry 2017, 56, 4154-4168.

(25) Reddi, A. R.; Pawlowska, M.; Gibney, B. R. Evaluation of the Intrinsic $\mathrm{Zn}$ (II) Affinity of a Cys3His1 Site in the Absence of Protein Folding Effects. Inorg. Chem. 2015, 54, 5942-5948.

(26) Hardy, R. W.; Wertz, G. W. The Cys(3)-His(1) motif of the respiratory syncytial virus M2-1 protein is essential for protein function. J. Virol. 2000, 74, 5880-5885.

(27) Cai, H.; Zhang, Y.; Ma, Y.; Sun, J.; Liang, X.; Li, J. Zinc binding activity of human metapneumovirus M2-1 protein is indispensable for viral replication and pathogenesis in vivo. J. Virol. 2015, 89, 6391405.

(28) Outten, C. E.; O'Halloran, T. V. Femtomolar sensitivity of metalloregulatory proteins controlling zinc homeostasis. Science 2001, 292, 2488-2492.

(29) Biasini, M.; Bienert, S.; Waterhouse, A.; Arnold, K.; Studer, G.; Schmidt, T.; Kiefer, F.; Cassarino, T. G.; Bertoni, M.; Bordoli, L.; Schwede, T. SWISS-MODEL: modelling protein tertiary and quaternary structure using evolutionary information. Nucleic Acids Res. 2014, 42, W252-W258.

(30) Bienert, S.; Waterhouse, A.; de Beer, T. A.; Tauriello, G.; Studer, G.; Bordoli, L.; Schwede, T. The SWISS-MODEL Repositorynew features and functionality. Nucleic Acids Res. 2017, 45, D313D319.

(31) Humphrey, W.; Dalke, A.; Schulten, K. VMD: visual molecular dynamics. J. Mol. Graphics 1996, 14, 33-38.

(32) Krieger, E.; Joo, K.; Lee, J.; Lee, J.; Raman, S.; Thompson, J.; Tyka, M.; Baker, D.; Karplus, K. Improving physical realism, stereochemistry, and side-chain accuracy in homology modeling: Four approaches that performed well in CASP8. Proteins 2009, 77, 114-122. 